\title{
A ROBUST FAULT DIAGNOSIS AND FORECASTING APPROACH BASED ON KALMAN FILTER AND INTERVAL TYPE-2 FUZZY LOGIC FOR EFFICIENCY IMPROVEMENT OF CENTRIFUGAL GAS COMPRESSOR SYSTEM
}

\author{
Bachir NAIL ${ }^{*, * *}$, Abdellah KOUZOU*,**, Ahmed HAFAIFA*,**, Nadji HADROUG ${ }^{*, * *}$, Vicenç PUIG $^{* * * *}$ \\ *Applied Automation and Industrial Diagnostics Laboratory, Faculty of Sciences and Technology, \\ University of Djelfa 17000 DZ, Algeria. \\ ${ }^{* *}$ Gas Turbine Joint Research Team, University of Djelfa, Djelfa 17000 DZ, Algeria \\ b.nail@univ-djelfa.dz, hafaifa.ahmed.dz@ieee.org, kouzouabdellah@ieee.org,n.hadroug@univ-djelfa.dz
} ${ }^{* * *}$ Automatic Control Department, Universitat Politècnica de Catalunya (UPC), TR11, Rambla de Sant Nebridi, 10, 08222 Terrassa, Spain, vicenc.puig@upc.edu

\section{Abstract}

The paper proposes a robust faults detection and forecasting approach for a centrifugal gas compressor system, the mechanism of this approach used the Kalman filter to estimate and filtering the unmeasured states of the studied system based on signals data of the inputs and the outputs that have been collected experimentally on site. The intelligent faults detection expert system is designed based on the interval type- 2 fuzzy logic. The present work is achieved by an important task which is the prediction of the remaining time of the system under study to reach the danger and/or the failure stage based on the Auto-regressive Integrated Moving Average (ARIMA) model, where the objective within the industrial application is to set the maintenance schedules in precisely time. The obtained results prove the performance of the proposed faults diagnosis and detection approach which can be used in several heavy industrial systems .

Keywords: Fault detection and diagnosis, Centrifugal gas compressor, Kalman filter, Interval Type-2 fuzzy logic, Experimental data, ARIMA

\section{Nomenclature}

AIC ARIMA

BCL 505

ACS

ESRIV

FDI

FL

FL-2

FPE

GCV

GE

HP

IV

LP

LNG

LSL

MIMO

OE

PLC

RMSE

SISO

SPC

SQC

SS

SRIV

USL

VAF

Symbols

$T 1$

$P 1$

$T 2 / \hat{T} 2$
Akaike Information Criterion

Auto-regressive Integrated Moving Average

Barrel, Closed disc counter wheel, Libre vortex diffuser, $500 \mathrm{~mm}$ wheels diameter with 5 wheels Acquisition Control System

Extended Simplified Refined Instrumental Variable Fault Detection and Isolation

Fuzzy Logic Type-1

Fuzzy Logic Type-2

Final Prediction Error

Gas Control Valve

General Electric

High Pressure

Instrumental Variable

Low Pressure

Liquefied Natural Gas

Lower Specification Limit

Multi Input Multi Output

Output Error

Programmable Logic Controller

Root Mean Square Error

Single Input Single Output

Statistical Process Control

Statistical Quality Control

State Space

Simplified Refined Instrumental Variable

Upper Specification Limit

Variance Accounting For

Aspiration Temperature $\left[{ }^{\circ} \mathrm{C}\right]$

Aspiration Pressure $[\mathrm{kg} / \mathrm{cm} 2]$

Discharge temperature/Observesd discharge temperature

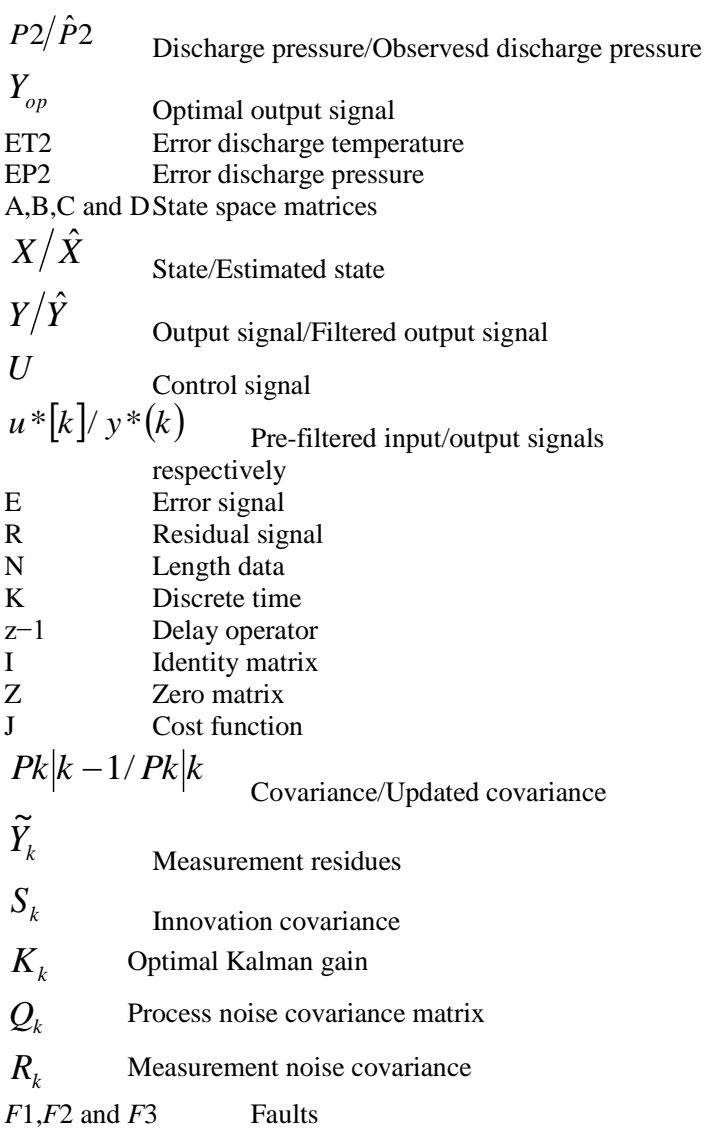




$\begin{array}{ll}S & \text { Standard deviation } \\ m & \text { Average value } \\ \tilde{F}_{2}^{j} & \text { Sets of premises } \\ \tilde{G}^{j} & \text { Sets of consequences } \\ \operatorname{col}\{.\} & \text { Transforms a matrix into a column vector } \\ & \text { Indicates estimated values } \\ n a & \text { The index of matrix polynomial } A\left(z^{-1}\right) \\ b n & \text { The index of matrix polynomial } B\left(z^{-1}\right) \\ P & \text { The number of the outputs signals } \\ \mathbf{G r e e k s} & \\ \otimes & \text { Kronecker product } \\ \theta / \hat{\theta} & \text { Matrix parameters/Estimated matrix parameters } \\ \Phi & \text { Matrix inputs/outputs information }\end{array}$

\section{INTRODUCTION}

In the last few years, the maintenance of industrial systems during their operating modes is one of the main strategic problems facing the industry, from the design of a machine until to its exploitation. Therefore, the diagnostic system is essential for ensuring the smooth and continuous operation of dynamic systems and for increasing their performances by guaranteeing better reliability. Indeed, the diagnostic system is used to provide the control system by the required real data of the dynamic system operating status in un-faulty (healthy) mode and in faulty mode. On the other side, the diagnostic system has to fulfill the requirement of robustness to avoid the practical cases of non-detection and false alarms, which means avoiding the eventual accidental and catastrophic situations. The diagnostic systems is based mainly on comparing the actual behavior of the system with a reference behavior representing the healthy operation. This comparison allows to detect the behavioral changes that are due to the appearance of the faults.

The FDI diagnosis approaches implemented in the industries are generally divided in two main classes such as the diagnostic approaches based on mathematical model and the diagnostic approaches based on data analysis. The FDI mathematical model approaches include, the observer's approach [1], the parity-space approach [2], and system identification-based approaches [3]. The FDI data analysis approaches or called the measurements approaches, these approaches insure the detection of fault under certain conditions, included the artificial intelligence-based model (fuzzy logic, neural networks...etc.) [4], statistical approaches and FDI approaches based on signal processing [5, $6]$.

In this sense, several works have been carried out in the literature presenting the main challenges of the proposed diagnosis approaches in energy plants, (gas turbine, gas compressor, centrifugal chiller, turbomachinery...etc.), such as fault detection and diagnostics tool based on a data fault library, application to an automated chiller [7],
Effect of common faults on the performance of different types of vapor compression systems [8], A statistical fault detection and diagnosis method for centrifugal chillers based on exponentiallyweighted moving average control charts and support vector regression [9], the performance diagnosis of gas turbine compressors based on component map tuning method [10], diagnostics of gas turbine for a high bypass ratio military turbofan engine based on artificial neural-networks [11], Active surge control for variable speed axial compressors [12], and fault-diagnoses based on evolution strategy (a simple genetic-based algorithm and fuzzy approaches) for gas turbocompressor systems $[13,14,15,16,17,18,19,20$, 21]. Based on these research works, it is obvious that the research on the gas turbine and gas compressor systems are actually a very highlighted research topic.

The proposed diagnostic approach presented in this paper is applied on the centrifugal gas compressor system which is used in many sectors and covers a very wide range of industrial applications. Indeed, this system is at the heart of many industrial sectors, such as the petroleum industry, the thermal and the nuclear power generation, the aeronautic and the space propulsion, the automobile industry, and the transport of gases (pipelines)[22, 23, 24, 25]. A good understanding of this system operation is essential issue for increasing their performance and reducing their operating costs. In this case, one of the limits of using this system is determined by the stability limits, where it is well known that beyond these limits the operation system stability cannot be ensured. In order to take into account the characteristics of the studied gas compressor system and its operating conditions, it is necessary to establish a dynamic model that covers all the dynamic behaviors. This dynamic model presents an important element in the proposed diagnosis and detection approach, where the main objective is to obtain the best dynamical model based on previous works. Indeed, several recent researches have been dealt with the gas compressor systems modeling such as: the industrial centrifugal compressors modeling based of fuzzy logic approach [26], the centrifugal gas compressor parametric modeling based on system identification [27], the two shaft gas turbine modeling based on linearized model[28], the system simulation for dynamic centrifugal compressor model [29], and others [30, 31, 32, 33].

According to the International Energy Agency, Algeria is ranked in the 10th position among the natural gas producers in 2015 with $2.3 \%$ of the total world production and in the 6 th position among the natural gas exporters with $5.3 \%$ of the world total production. Recently with the emergence of new natural gas fields, Algeria is expected to increase its natural gas network production capacity by nearly 35 billion cubic meters by 2020 , this is guided by 
the evolution of needs expressed by the global gas market and the emergence of new sources discovered by the Algerian company Sonatrach.

Therefore, the development of the interconnection capacity of the gas transmission network is in function of gas compression stations installed in the hydrocarbon field, requires very complex equipment, distributed over several stations of gas compression along the pipeline, to distribute the gas to different users. The most suitable machines are gas centrifugal compressors, these machines are subject to several problems of operation instability, causing oscillations that can be dangerous for their mechanical strength causing a total or a partial degradation of such machines.

The novelty of this work consists in proposing a new approach for faults detection in a centrifugal gas compressor to ensure its safety operation, its availability for production and to minimize its maintenance cost. This approach is applied to a BCL 505 centrifugal gas compressor used by Sonatrach in the Hassi R'Mel gas compressor station in the south of Algeria.

The main contribution of this paper focuses on the development of a robust faults diagnosis and detection approach which aims to increase the system monitoring performance during the operating mode of the studied centrifugal gas compressor plant. This proposed approach is based on the combination of the two FDI diagnosis model approaches (based on the parametric identification model and on observer system), with the intelligent expert fuzzy type-2 system as shown in Fig. 1.

The Kalman observer is used to estimate on real-time the filtered and unmeasured outputs $\hat{Y}$ (the discharge pressure and the discharge temperature $\mathrm{P} 2$, T2 respectively) of the centrifugal gas compressor system based on the two actual measured inputs (the temperature and the pressure aspiration $\mathrm{T} 1, \mathrm{P} 1$ respectively)). It important to clarify that the Kalman observer presented in this paper is built based on the experimental data of the inputs (T1 and P1) and the outputs (T2 and P2) obtained by the installed sensors via the acquisition control system (ACS). The equivalent model (healthy or un-faulty model) is obtained from parametric system identification based on the extended simplified refined instrumental variable (ESRIV) algorithm using experimental data [34]. This obtained optimal model is considered as a reference model which generates the reference outputs $Y_{o p}$, where the difference between the estimated outputs $\hat{Y}$ and the reference outputs $Y_{o p}$ presents the residues vector $r$. On the other side, an expert system is proposed and designed based on the type-2 fuzzy theory [35, 36], taking into account the faults detection threshold as proposed in [4]. This expert system receives the residues vector and generates the signature which provides the information about the fault occurrence. When a fault is occurred, it is important to estimate the remaining time before the studied system falls into failure, therefore a remaining time predictor based on the autoregressive integrated moving average (ARIMA) model is proposed in this paper to accomplish the whole proposed faults diagnosis and detection approach, where the main aim is to predict the remaining time starting from the instant of failure occurrence to the estimated final stop instant [37, 38].

\section{HASSI R'MEL GAS FIELD}

Hassi R'Mel gas field is located approximately $550 \mathrm{~km}$ south of Algiers (Algeria), at an altitude of $760 \mathrm{~m}$. This gas field covers an area of $3500 \mathrm{~km} 2$, $70 \mathrm{~km}$ in the north-south direction and $50 \mathrm{~km}$ in the east-west direction. The landscape is composed of a vast rocky plateau, the climate is characterized by an average humidity of $19 \%$ in summer and $34 \%$ in winter. The temperature has a large range variation between $0{ }^{\circ} \mathrm{C}$ in winter to $45^{\circ} \mathrm{C}$ in summer. The production of the Hassi R'Mel gas field can reach the capacity of 100 billion cubic meters of natural dry gas, 12 million tons of condensate gas and 3.5 million tons of liquefied natural gas (LNG), The essential components of the LNG are expressed in Fig. 2, these components are presented following their molar fractions (blue color) and molecular weights (green color).

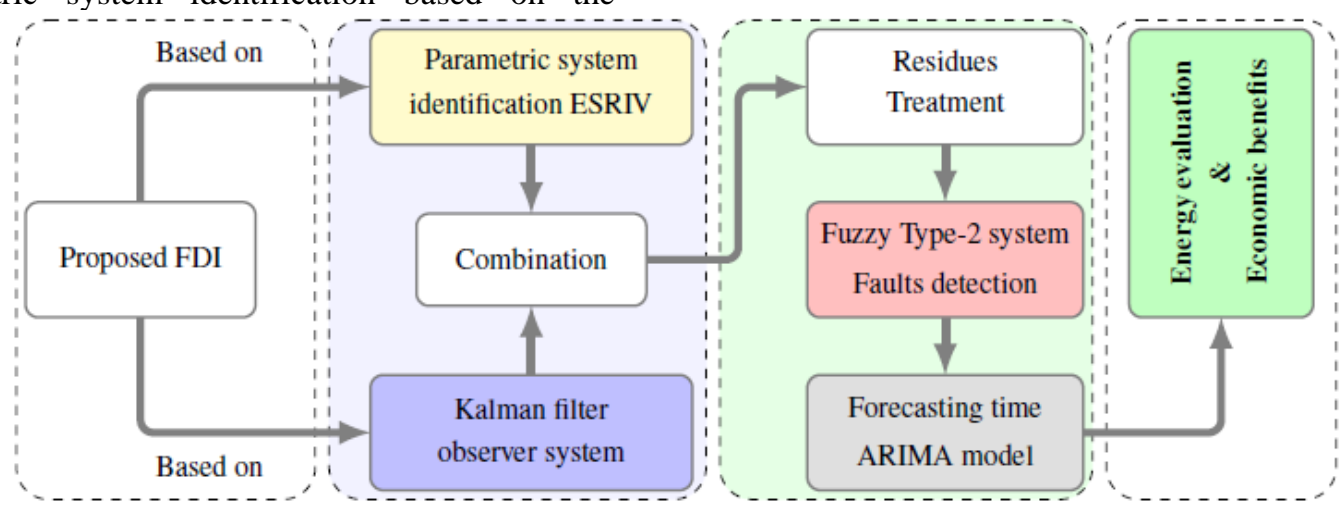

Fig. 1. Proposed FDI approach 

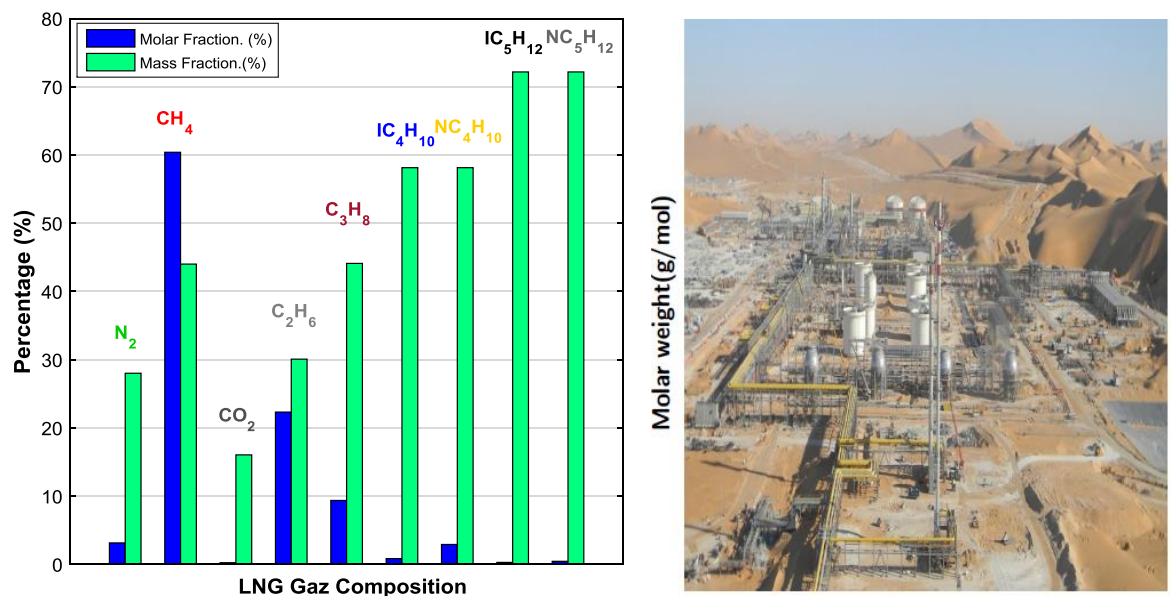

Fig. 2. Characteristics and components of LNG

Hassi R'Mel gas field consists of three main exploitation zones, the north zone, the south zone and the center zones, each zone contains many pumping centrals, gas treatment factories, oil stations and gas compression stations, this last contains the centrifugal gas compressor which in the research purpose application of the proposed faults diagnosis and detection approach proposed in this paper. The main role of the gas compression stations is to constantly pressurize the dry gas to maintain its required pressure level. to regulate the gas pressure at the national level and the international market level. Each station contains 18 turbo gas compressor (Centrifugal gas compressor driven by a gas turbine (GEMS5002C)) as shown in Fig. 3, each pair of turbo-compressor forms a compression line. The compression process is carried out through two stages, the low pressure stage and the high pressure stage, where a cooling system based on air coolers is used to regulate the temperature at the intermediary of the two stages.

\section{CENTRIFUGAL GAS COMPRESSOR BCL 505 SYSTEM}

Centrifugal compressors are used in many industrial sectors, such as the oil industry, the production of thermal and nuclear energy, aerospace propulsion, automotive, water distribution, etc. Indeed, a good understanding of the operation of these devices is essential to increase their performance and reduce their operating cost. In this case, one of the limits of use of these systems is determined by its stability limits, limits beyond which stable operation of systems is no longer ensured. The centrifugal gas compressor studied in this paper is the BCL 505 type which is shown in Fig. 4, its characteristics are presented in appendix A Table 3.

This compressor is constructed by Nuovo Pignone company and it is used in a gas compression heavy application such as in gas field production and gas network transportation, it is equipped with a control room computer-based where the DCS is a part of it which allows to take directly inputs / outputs measurements from the installed sensors. The main function of the studied centrifugal gas compressor in this paper, is to ensure the pressure rise of the continuous flow of gas passing through it based on kinetic energy. Where The increase of the gas pressure by a compressor is used to:

$\checkmark$ reach a level of gas pressure.

$\checkmark$ compensate the pressure losses related to the circulation of the gas flow in a gas network.

The compressors can be classified according to their characteristics depending on the type of gas to be compressed such as, air compressors and gas compressors, and/or depending on the movement of the moving parts such as, linear or rotary motion, and/or depending on the operating principle such as, volumetric compressors and dynamic compressors which are the application area of the present paper.

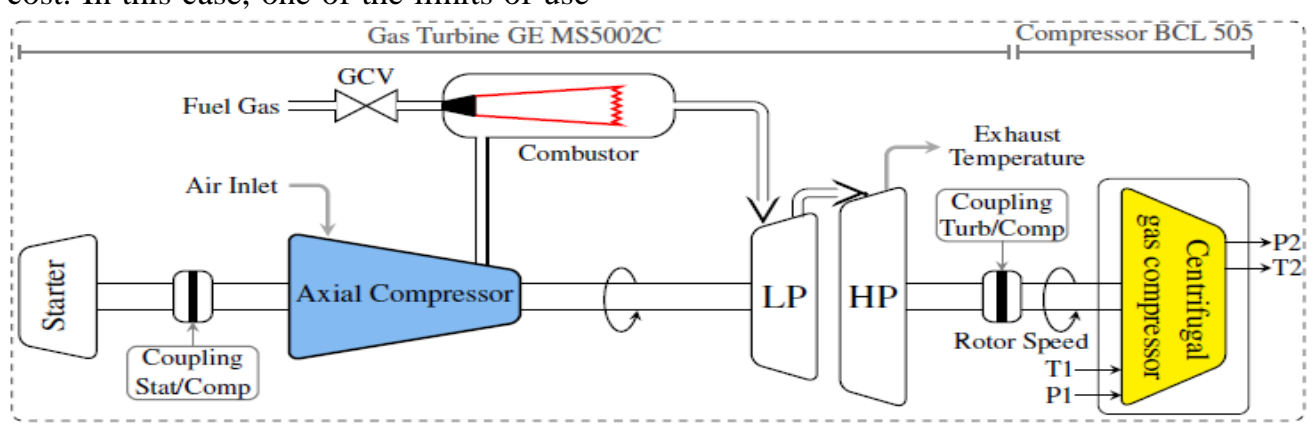

Fig.3. Schematic block diagram of Turbo-compressor system 


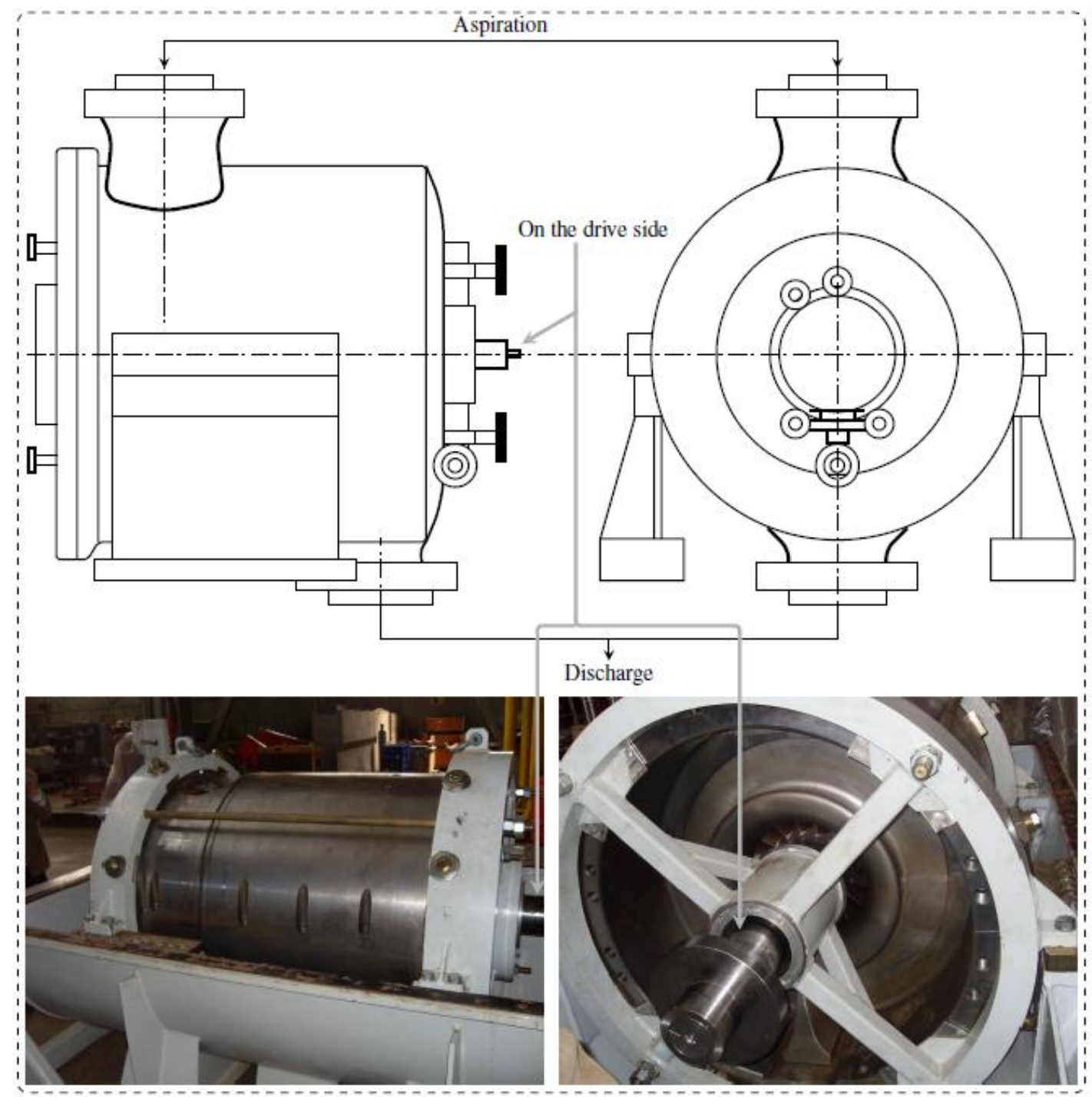

Fig. 4. Centrifugal gas compressor BCL 505 body, with real view in vertical joint plane

\section{PARAMETRIC MODELING OF DYNAMIC CENTRIFUGAL GAS COMPRESSOR BCL 505 SYSTEM}

The single input single output (SISO) simplified refined instrumental variable (SRIV) algorithm is dedicated for solving the problem of parameters estimation of the output error (OE) model which is presented as follows [34]:

$$
y[k]=\frac{B\left(z^{-1}\right)}{A\left(z^{-1}\right)} u[k]+e[k]
$$

To ensure the error minimization, the least squares cost function is used, it is expressed as follows:

$$
J=\sum_{k=1}^{N} \hat{e}[k]
$$

Where $\hat{e}[k]$ is the estimated error function obtained directly by inspection of the model based on equation (1), $\hat{e}[k]$ can be expressed as follows:

$$
\hat{e}[k]=y[k]-\frac{\hat{B}\left(z^{-1}\right)}{\hat{A}\left(z^{-1}\right)} u[k]
$$

This error function contains the unknown parameters of the polynomials. Furthermore, it can be rewritten as follows:

$$
\hat{e}[k]=\frac{1}{\hat{A}\left(z^{-1}\right)}\left\{\hat{A}\left(z^{-1}\right) y[k]-\hat{B}\left(z^{-1}\right) u[k]\right\}
$$

This equation can be furthermore simplified:

$$
\hat{e}[k]=\hat{A}\left(z^{-1}\right) y^{*}[k]-\hat{B}\left(z^{-1}\right) u^{*}[k]
$$

Where $y^{*}[k]$ and $u^{*}[k]$ are the pre-filtered signals defined as follows:

$$
\begin{aligned}
& y^{*}[k]=\frac{1}{\hat{A}\left(z^{-1}\right)} y(k) \\
& u^{*}[k]=\frac{1}{\hat{A}\left(z^{-1}\right)} u(k)
\end{aligned}
$$


Equation (5) is a linear transfer function model, so that the normal Instrumental Variable (IV) methods can be used to estimate the parameters if it is possible to perform the pre-filtering operations in (6) and (7). The parameters of $A\left(z^{-1}\right)$ are axiomatically unknown and so this pre-filtering operation can be adaptive [34]. For MIMO system equation (1) can be rewritten as follows:

$$
y[k]=A^{-1}\left(z^{-1}\right) B\left(z^{-1}\right) u[k]+e[k]
$$

Where,

$$
\left\{\begin{array}{l}
A\left(z^{-1}\right)=I_{p}+A_{1} z^{-1}+\Lambda+A_{n a} z^{-n a} \\
B\left(z^{-1}\right)=B_{0}+B_{1} z^{-1}+\Lambda+B_{n b} z^{-n b}
\end{array}\right.
$$

based on $A\left(z^{-1}\right)$ and $B\left(z^{-1}\right)$ expansions and equation (8), the following equation is obtained:

$$
\begin{aligned}
& A\left(z^{-1}\right) e[k]=y[k]+A_{1} y[k-1] \Lambda+ \\
& A_{n a} y[k-n a]-B_{1} u[k-1] \Lambda+B_{n b} u[k-n b]
\end{aligned}
$$

Equation (9) can be rewritten using the Kronecker operator as:

$$
\begin{aligned}
& A\left(z^{-1}\right) e[k]=\left[I_{p} \otimes y[k]^{T}\right] \operatorname{col}\left(I_{p}\right) \\
& +\left[I_{p} \otimes y[k-1]^{T}\right] \operatorname{col}\left(A_{1}^{T}\right) \Lambda \\
& +\left[I_{p} \otimes y[k-n a]^{T}\right] \operatorname{col}\left(A_{n a}^{T}\right) \\
& -\left[I_{p} \otimes u[k-1]^{T}\right] \operatorname{col}\left(B_{1}^{T}\right) \Lambda \\
& -\left[I_{p} \otimes u[k-n b]^{T}\right] \operatorname{col}\left(B_{n b}^{T}\right)
\end{aligned}
$$

Finally, the error $e[k]$ can be expressed as follows:

$$
\begin{aligned}
& e[k]=A^{-1}\left(z^{-1}\right)\left[I_{p} \otimes y[k]^{T}\right] \operatorname{col}\left(I_{p}\right)+ \\
& {\left[A^{-1}\left(z^{-1}\right)\left[I_{p} \otimes y[k-1]^{T}\right] \Lambda+A^{-1}\left(z^{-1}\right)\left[I_{p} \otimes y[k-n a]^{T}\right]\right.} \\
& -A^{-1}\left(z^{-1}\right)\left[I_{p} \otimes u[k-1]^{T}\right] \Lambda-A^{-1}\left(z^{-1}\right)\left[\begin{array}{c}
\operatorname{col}\left(A_{1}^{T}\right) \\
\mathrm{M} \\
\operatorname{col}\left(A_{n a}^{T}\right) \\
\operatorname{col}\left(B_{1}^{T}\right) \\
\mathrm{M} \\
\operatorname{col}\left(B_{n b}^{T}\right)
\end{array}\right]
\end{aligned}
$$

Consequently,

$$
e[k]=A^{-1}\left(z^{-1}\right)\left|I_{p} \otimes y[k]^{T}\right| \operatorname{col}\left(I_{p}\right)-\Phi^{T}[k] \theta
$$

The centrifugal gas compressor dynamical model can be obtained using the ESRIV algorithm, its main steps are summarized in appendix B.

The BCL 505 centrifugal gas studied in this paper is a MIMO system with two inputs, the aspiration temperature $T l$ and the aspiration pressure $P l$ and two outputs, the discharge pressure $P 2$, and the discharge temperature $T 2$. The data used in this modeling is obtained experimentally on site, it contains $N=1208$ samples along a duration of 1208 hours, it is important to clarify that this experimental data is obtained via several tests to get the optimal data that are covering all possible dynamic behavior of this centrifugal gas compressor.

The application of the proposed ESRIV algorithm based on experimental data allows to obtain the best model of the studied system, the algorithm converges totally only in five iterations following the proposed flowchart. Fig. 5 presents the validation criteria values function of the number of iterations. It is obvious that the best model is corresponding to the iteration number five where all the criteria reach their minima in comparison with the other iterations.

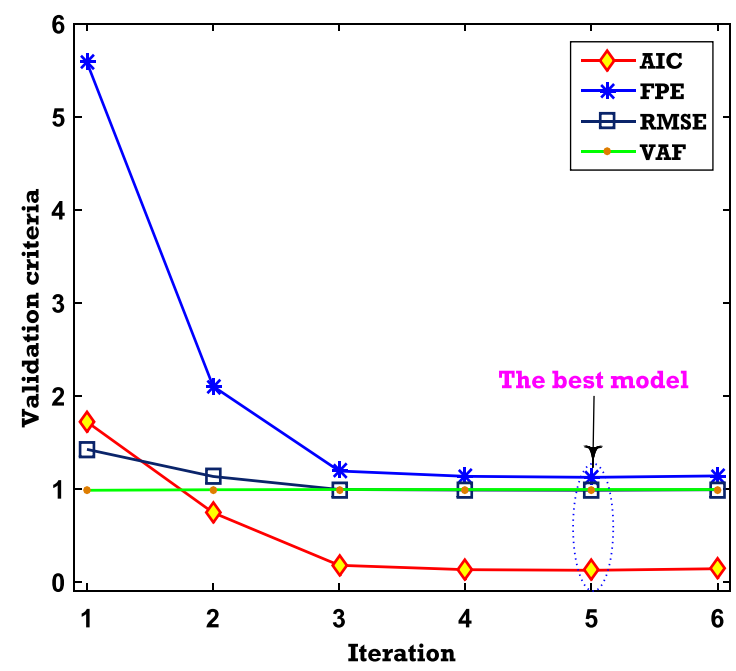

Fig. 5. Validations criteria of the dynamic model of centrifugal gas compressor

The convergence of the two outputs (the discharge pressure $\mathrm{P} 2 /$ and the discharge temperature $\mathrm{T} 2$ ), that are obtained by the identification from the best selected model are compared with the real output measurements obtained experimentally on site, as shown in the following figures Fig. 6 and Fig. 7.

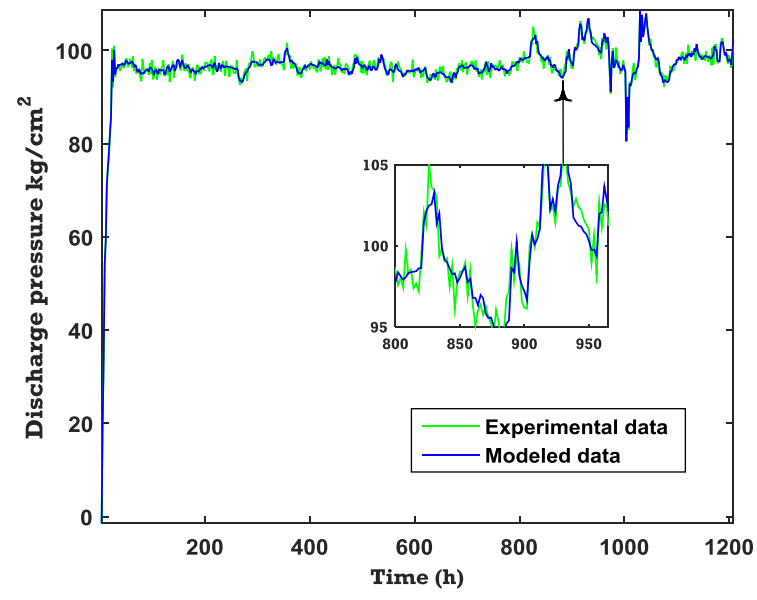

Fig. 6. Output signal of experimental and modeled discharge pressure 


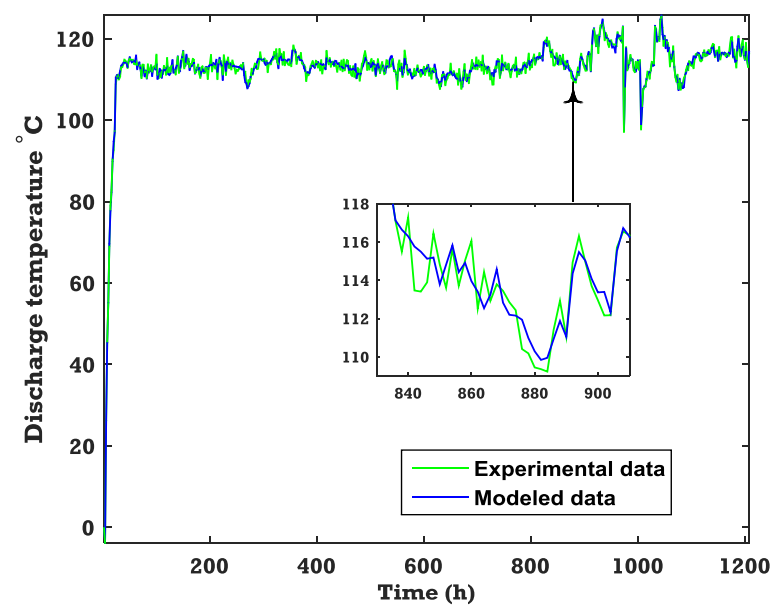

Fig. 7: Output signal of experimental and modeled discharge temperature

The centrifugal gas compressor dynamic model can be represented by a transfer function matrix in discrete-time $G\left(z^{-1}\right)$ which is containing five coefficient matrices $A_{1 \Lambda 5} \in \mathfrak{R}^{2 \times 2}$ in denominator, and five coefficient matrices $B_{1 \Lambda 5} \in \mathfrak{R}^{2 \times 2}$ in numerator.

$$
G\left(z^{-1}\right)=\frac{B\left(z^{-1}\right)}{A\left(z^{-1}\right)}
$$

Where ,

$\left\{\begin{array}{l}A\left(z^{-1}\right)=A_{1} z^{-1}+A_{2} z^{-2}+A_{3} z^{-3}+A_{4} z^{-4}+A_{5} z^{-5} \\ B\left(z^{-1}\right)=B_{1} z^{-1}+B_{2} z^{-2}+B_{3} z^{-3}+B_{4} z^{-4}+B_{5} z^{-5}\end{array}\right.$ and,

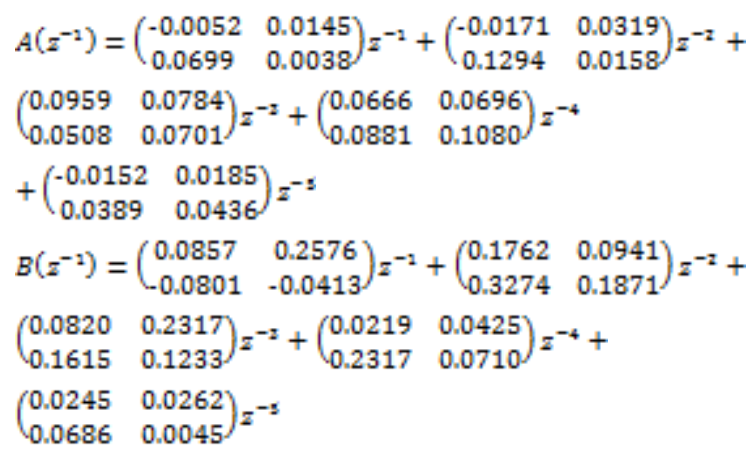

\section{THE POWER AND THE EFFICIENCY OF THE STUDIED SYSTEM}

The performance evaluation of a compressor is generally not limited to a single operating point, it is characterized by a field as shown in Fig. 8 The compressor field shows the compression ratio as a function of the corrected flow at a constant corrected rotation speed, to whom the iso-efficiency contours are often superimposed.

The limit at low flow rates is the pumping, which is characterized by the flow instability (sometimes until flow reversal), accompanied by pressure oscillations of great amplitude, which can eventually damage the machine. At high flows, the limit is the blockage, which corresponds to the appearance of a vibration phenomenon as sonic section in the floor.

These are considered as global efficiencies because they only take into account the initial and final states of the transformation. On the other hand, they are also considered as energetic efficiencies because they are related to the total enthalpy changes $h 0$ (they are called, for this reason, total-to-total efficiencies). These efficiencies can be used only if there is an exchange of energy. Using the same notations used previously in the (thermal) enthalpy diagrams, according to the second principle of thermodynamics as shows in Fig. 9, the total-to-total isentropic efficiencies of a compressor moving wheel can be defined as follows:

$$
\eta_{p . i}=\frac{h_{02 s}-h_{01}}{h_{02}-h_{01}}
$$

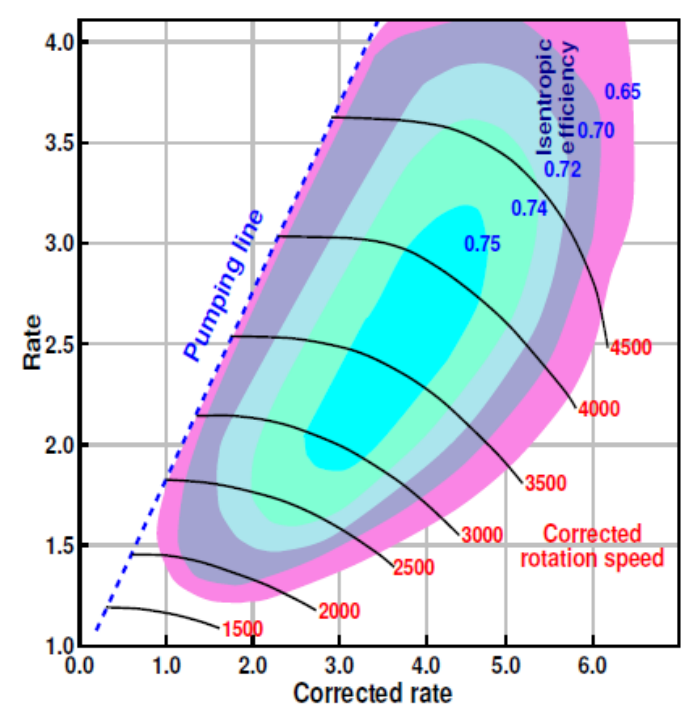

Fig. 8. The centrifugal gas compressor system field

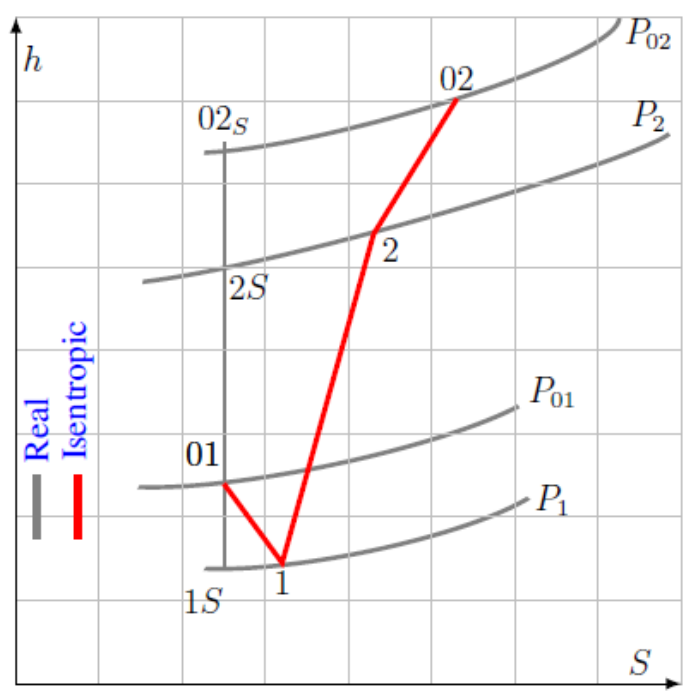

Fig. 9. Diagram efficiency thermodynamics of the studied system 
Figures Fig. 10 and Fig. 11 represents the produced power by the centrifugal gas compressor and its efficiency under the healthy operation state respectively, where the variation in the produced power (increase/decrease) depends on the market demand.

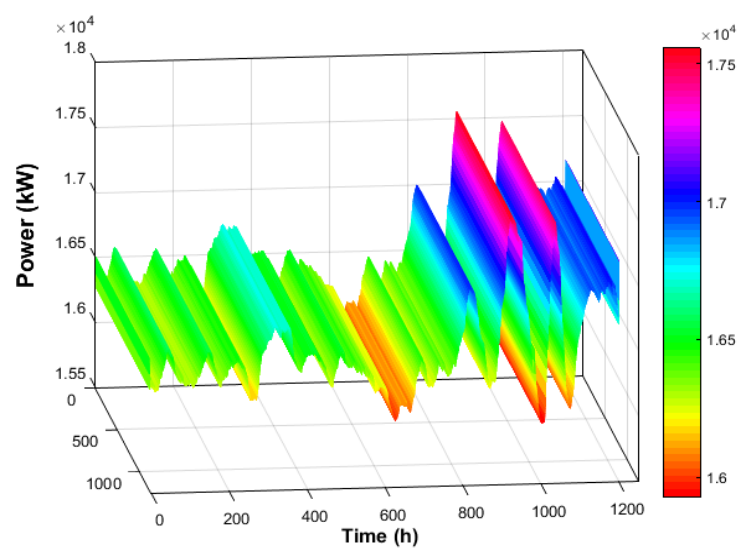

Fig .10. The hourly evolution of the power of the centrifugal gas compressor system

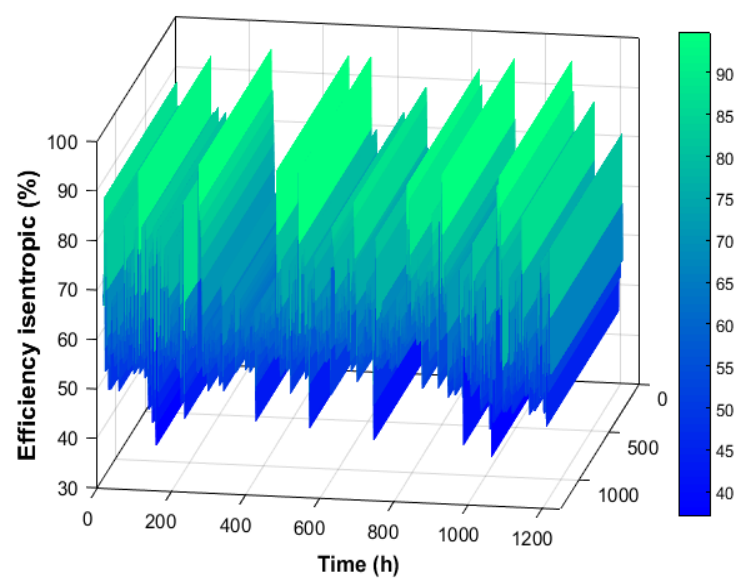

Fig. 11.The hourly evolution of the efficiency of centrifugal gas compressor system

\section{OUTPUTS ESTIMATION BASED ON KALMAN FILTER OBSERVER}

The Kalman filter is a set of mathematical equations which provides an efficient recursive computational algorithm to ensure the estimation of dynamic systems outputs states, in a way to minimize the mean of the squared error between the estimated output values and the real or the measured outputs. This filter is very useful in several domains: robotic motion planning, signal processing and econometrics. It can achieve the estimations of the past, the present, and even the future states. the last estimation which is dedicated to the future states is the main target of the work presented in this paper.

The dynamic model of the centrifugal gas compressor presented in equation (15), can be written under the block observable state space (SS) representation as follows :

$$
\left\{\begin{array}{l}
X(k+1)=A X(k)+B U(k) \\
Y(k)=C X(k)
\end{array}\right.
$$

Where, $A \in \mathfrak{R}^{10 \times 10}$ is the system matrix, $B \in \mathfrak{R}^{10 \times 2}$ is the controllability matrix, $C \in \mathfrak{R}^{2 \times 10}$ is the observability matrix, $X \in \mathfrak{R}^{10 \times 1}$ is the state vector, $U=[P 1, T 1]^{T} \in \mathfrak{R}^{2 \times 1}$ is the input vector and $Y=[P 2, T 2]^{T} \in \mathfrak{R}^{2 \times 1}$ is the output vector. The Kalman Filter observer algorithm is used to estimate the unmeasured states and the two filtered outputs $\hat{Y} \in \mathfrak{R}^{2 \times 1}$ of the centrifugal gas compressor, the discharge pressure $\hat{P}_{2}$ and the discharge temperature $\hat{T}_{2}$ respectively, The algorithm steps of the Kalman filter are presented in [6]:

From these results, it can be concluded that the proposed Kalman filer allows to obtained an accurate estimated output $\hat{Y}$ for the discharge pressure $P_{2}$ and the discharge temperature $T_{2}$ in comparison with the experimental measured outputs $Y$. Based on the zoom zones presented in Fig. 12 and Fig. 13, it can be said that the residues presenting the deviation between the two outputs respectively are neglected and the designed Kalman filter can be an adequate observer for the applications presented in this paper. This observer is used as essential and important part of the proposed faults diagnosis and detection approach which is applied to the centrifugal gas compressor presented in this paper.

\section{PRELIMINARY CONCEPTS ABOUT TYPE-2 FUZZY LOGIC}

Initially, the concept of the fuzzy type- 2 set was introduced by the founder of the fuzzy logic Lot $€$ Zadeh [34, 35] as an extension of the concept of the fuzzy type-1 set. .e fuzzy type-2 set is characterized by a fuzzy membership function, that is, the degree of belonging of each element of the set is itself a fuzzy set in $[0,1]$. Such sets are advisable in the case where there is an uncertainty at the level of the value of the membership itself. .e uncertainty can be either in the form of the membership function or in one of its parameters. .e transition from an ordinary set to a fuzzy set is the direct consequence of the indeterminism of the value belonging to an element by 0 or 1 . Similarly, when the functions of an element belonging to fuzzy numbers cannot be determined in real numbers within $[0,1]$, then the fuzzy sets type-2 is used. For this purpose, the fuzzy sets type-1 can be considered as an approximation of the €rst order of uncertainty and the fuzzy sets type- 2 as a second-order approximation. 

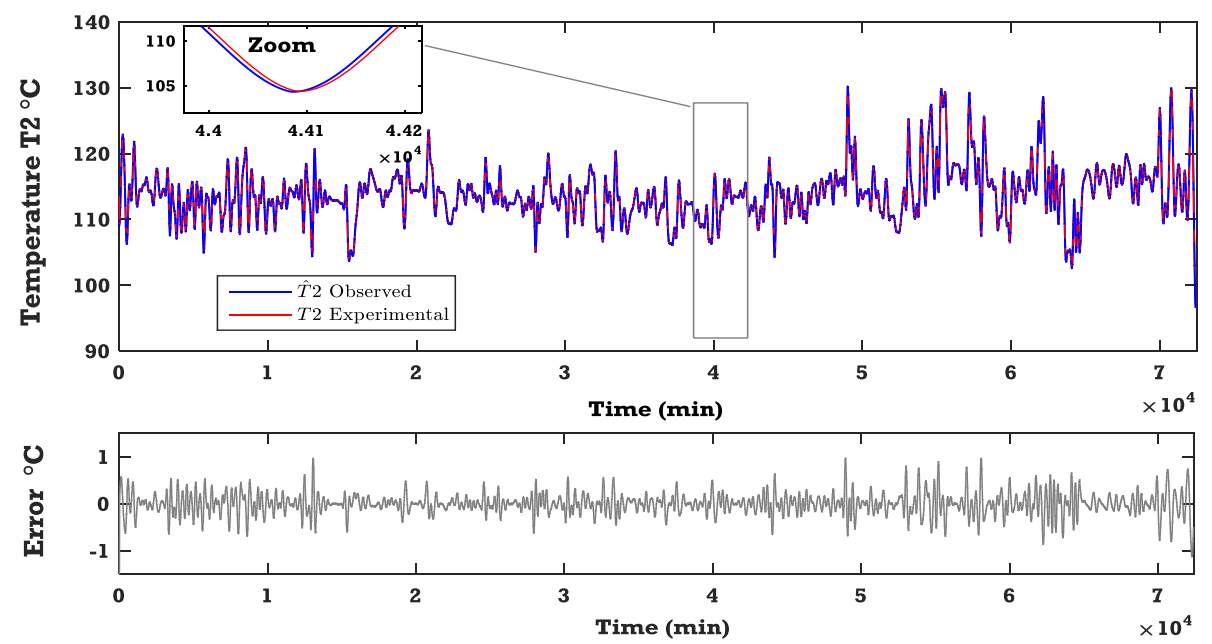

Fig. 12. Observed and experimental discharge temperature, and the error between them

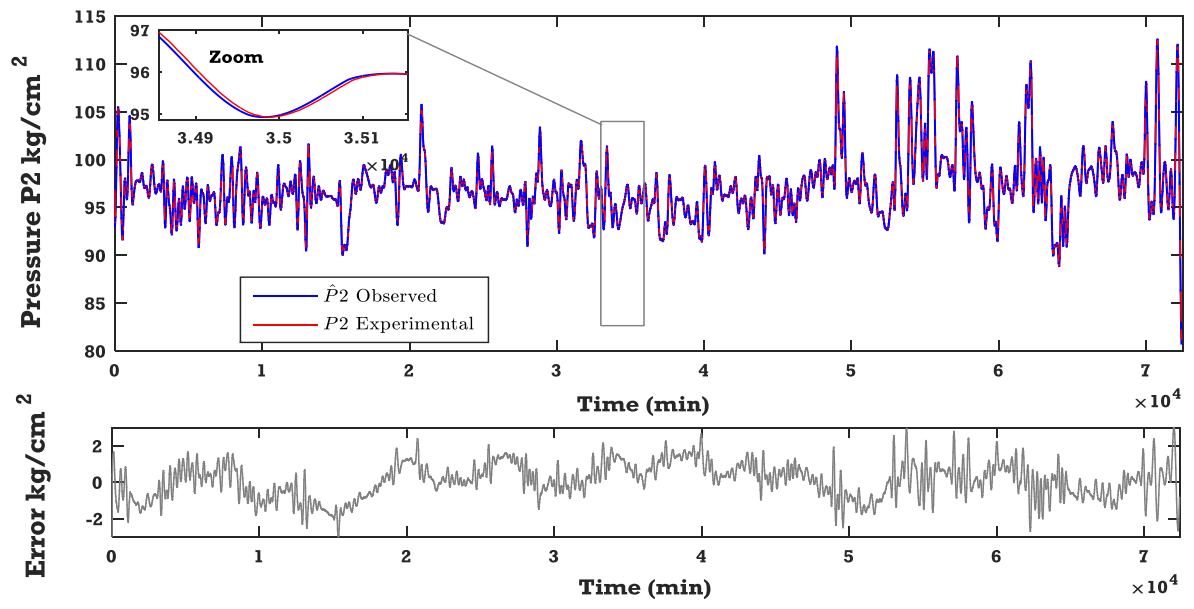

Fig. 13. Observed and experimental discharge pressure, and the error between them

Depending on the form of primary membership, there are many types of fuzzy sets type-2, among them: triangular, interval, gaussian, and the gaussian 2 which is used in the fuzzy type- 2 system faults detection in this paper.

The structure of a fuzzy type- 2 system is represented in Fig. 14, it is similar to fuzzy type-1. whereas the $€$.h block appears in the output processing is of the reduction type.

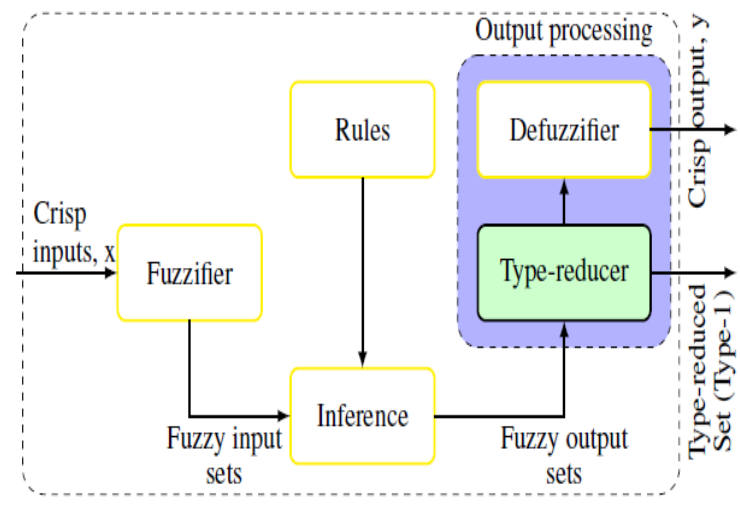

Fig. 14. The structure of the type-2 Fuzzy Logic System.
The difference between the rules of FL and FL2 , reside only in the nature of the membership functions. Therefore, the structure of the rules in the case of FL-2 will remain exactly the same as that of FL. The only difference is that some (or all) membership functions will be of FL-2. therefore, the $j^{\text {th }}$ rule of a FL-2 system will have the following form $[39,35,36]$ :

$$
\begin{aligned}
& \text { If } x_{1} i s \tilde{F}_{1}^{j} \text { and } x_{2} i \text { is } \tilde{F}_{2}^{j} \Lambda \text { and } x_{p} i s \tilde{F}_{p}^{j} \\
& \text { Then yis } \tilde{G}^{j}
\end{aligned}
$$

Where, $x_{1} \in X_{1}, x_{2} \in X_{2}, \mathrm{~K}, x_{p} \in X_{p}$ are the outputs, the $F_{1}^{j}$ are the sets of premises such as $i=1,2, \mathrm{~K}, p, y \in y$ is the output, and the $\tilde{G}^{j}$ are the sets of consequences. 


\section{THE PROPOSED FDI SETUP FOR A CENTRIFUGAL GAS COMPRESSOR PLANT}

The faults diagnosis and detection approach proposed in this research paper takes into consideration all the phases of the operating cycle of the centrifugal gas compressor system under study, such as the evolution of the pressure and the temperature of the gas in the centrifugal gas compressor, the evolution of mass and volume flows as function of the gas pressure and temperature. In the same time, it takes into account the power absorbed as a function of the gas, and other operating conditions that allow to obtain precise information about the dynamic behavior and to maximize the availability of this system. In this proposed diagnostic system a hybrid between the mathematical theory of estimation based on the stochastic kalman filter observer, and the artificial intelligence algorithms based on a type- 2 fuzzy system. Fig. 15, shows the proposed faults diagnosis and detection approach details studied presented in this paper.

On the other side, it is well known that the industrial systems have complex behaviors, and they are characterized by uncertain variables or parameters as a function of time, this constraint complicates their control task and implies many difficulties in achieving the good performances of such systems. For this purpose, this work proposes a real-time faults diagnosis and detection approach, where the main aim is to detect and to localize the defective components in the studied centrifugal gas compressor system.

This proposed approach is based on the calculation of the residues $\mathrm{r}(\mathrm{k})$ following equation (17), which presents the errors between the optimal Yop and the observed $\mathrm{Y}^{\wedge}$ outputs respectively. On the other side, the residues are the inputs for the fuzzy type-2 system, when the system is under healthy operating state, these residues have generally a null average and a determined variance. In practice, the residues do not have exactly zero value in the absence of faults as shown in the previous figures Fig. 12 and Fig. 13, because the obtained model of the studied system in this paper does not take into account all the internal and external parameters, which means that only the preponderant parameters are taken into account and that certain simplification has been considered. On the other side the measurements performed on the system are often affected by measurement noise. The residues are expressed as follows:

$$
r(k)=Y_{o p}(k)-\hat{Y}(k)
$$

Where, $r(k)=\left[r_{p}(k), r_{T}(k)\right]^{T} \in \mathfrak{R}^{2 \times 1}$.
In this context, an elementary detection method consists in comparing the value of the obtained residues with a predefined threshold (modeling errors function). An alarm is triggered each time this threshold is crossed:

$$
\left\{\begin{array}{l}
r(k) \leq \varepsilon \Leftrightarrow d(k)=0 \\
r(k) \phi \varepsilon \Leftrightarrow d(k) \neq 0
\end{array}\right.
$$

Where, $\mathrm{d}($.$) presents the vector of the faults.$

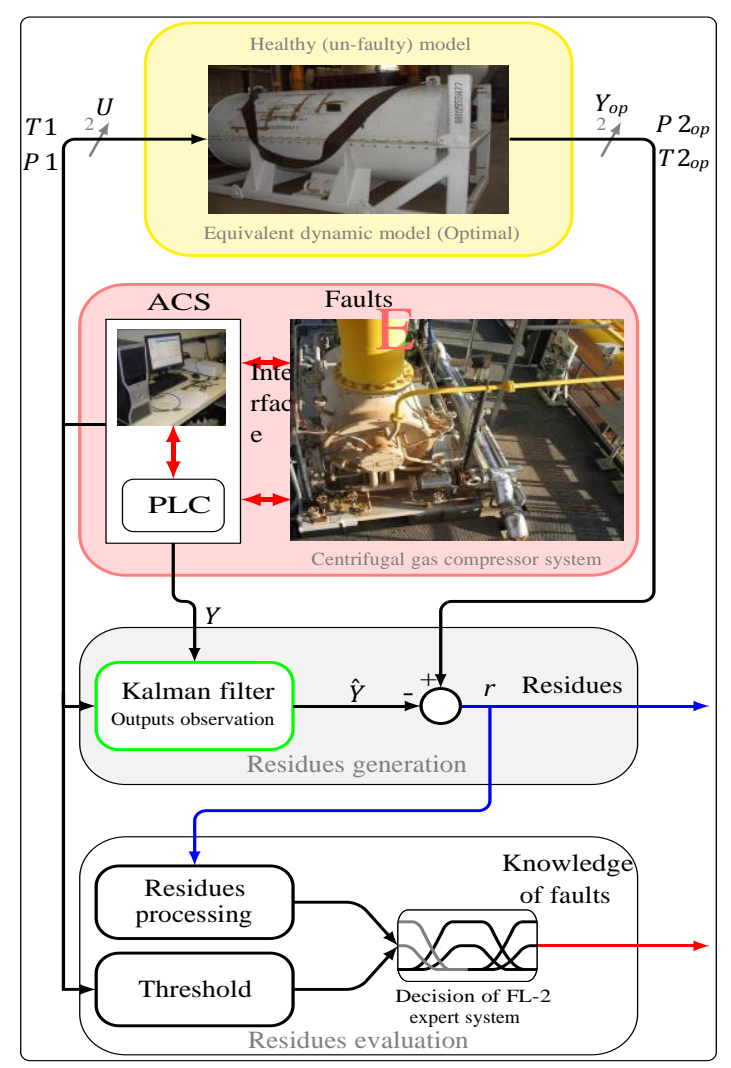

Fig. 15. Real-time faults detection diagnosis configuration for centrifugal gas compressor.

\subsection{Fault detection and isolation (FDI)}

The purpose of the detection procedure is to determine the instant of fault occurrence. To achieve this objective, the residues obtained by comparing the system optimal model outputs with the system estimated outputs.

In the presence of faults, the evolutions of the discharge temperature $\mathrm{T} 2$ and the discharge pressure P2 during the time interval of $7 \times 104$ minutes are registered via the DCS, where a rising vibration in the discharge temperature $\mathrm{T} 2$, and in the discharge pressure $\mathrm{P} 2$ are remarked, comparison between the outputs dynamic behavior of the centrifugal gas compressor with and without faults are shown in Fig. 16 and Fig. 17.

After the residues generation step, the next task is the detection of faults based on the obtained residues signals. In the present work, to ensure a 
robust faults diagnosis and defection, the statistical process control (SPC) is integrated in the monitoring of centrifugal gas compressor. This process control was introduced by Walter Shewhart [4], and it occupies a prominent place among the statistical quality control (SQC) tools. It includes a set of statistical methods to monitor and improve a production process.

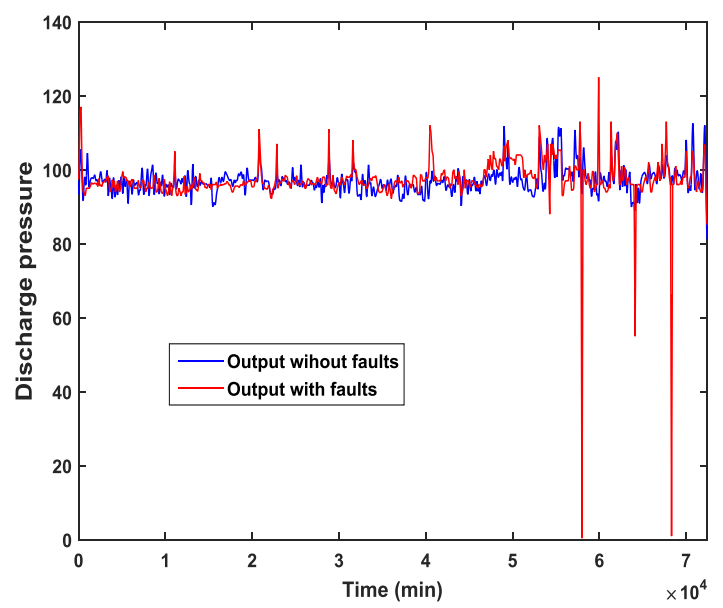

Fig. 16. The discharge pressure $\mathrm{P} 2$ with and without faults

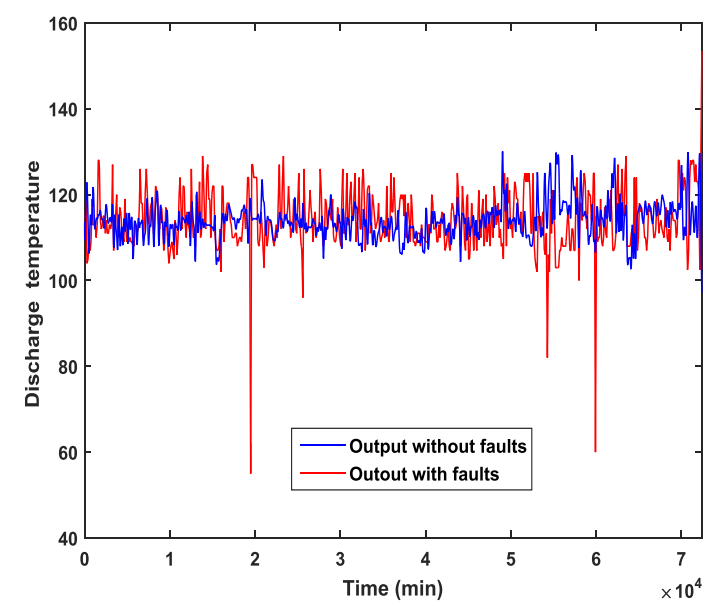

Fig. 17. The discharge temperature T2 with and without faults

The Shewhart graph is able to control the distribution of deviations instead of trying to control each individual deviation. The horizontal axis presents the time and the vertical axis presents the quality scale. It also contains three horizontal lines: The middle line presents the reference line of the normal operation mode output, the upper line is the upper specification limit (USL) of the control quality, and the lowest line is the lower specification limit (LSL) of the minimum control quality. When decisions are confined between the upper and lower limits, the deviation is acceptable and the centrifugal gas is operating in normal conditions. The following Table 1 presents the average value $\mathrm{m}$, standard deviation $\mathrm{s}$, and the
(USL,LSL) of the two outputs signal that are calculated based on Shewhart algorithm [4].

Fig. 18 and Fig. 19, show the variation of the generated residues of the discharge pressure and the discharge temperature respectively, that are included within the upper and lower detection faults lines (USL,LSL) of Shewhart graph for the two outputs of the studied centrifugal gas compressor system.

Table.1. The threshold faults detection

\begin{tabular}{cccc}
\hline Error & $m$ & $s$ & (USL,LSL) \\
\hline$E T 2$ & -0.0015 & $3.3063 \mathrm{e}-8$ & \pm 7.2755 \\
$E P 2$ & 0.0014 & $3.7597 \mathrm{e}-9$ & \pm 21.631 \\
\hline
\end{tabular}

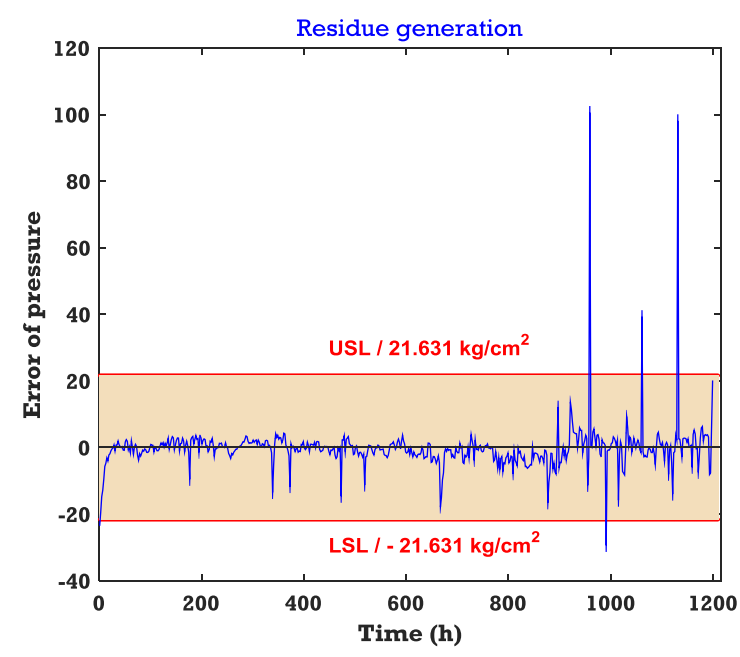

Fig. 18. The detection faults of $(P 2)$ with respect to a threshold

When the residues exceed the upper and lower allowed limits (threshold), the system operates with faults that may not appear externally at the beginning. Therefore, the problem which needs to be solved is to find an efficient way which allows to indicate immediately the faults when they are occurred. To solve this problem a type-2 fuzzy logic system is suggested as an expert model in order to detect and identify the type of occurring faults. In this case many tests have been done to select the best fuzzy sets type-2 that gives a robust performance and good results, in this work the gaussian 2 membership function was chosen as the best one.

Fig. 20, shows the expert model detection of the discharge pressure P2 which contains three membership functions of type gaussian 2 , that are the small, the medium, and the large with identical intervals between the upper- and lower-lines faults detection presented in Table 1, if there are faults, the expert model indicates "1". Fig. 21, shows the 
detection expert model of the discharge temperature $\mathrm{T} 2$, it differs from the first one only by the faults detection upper and lower lines (threshold).

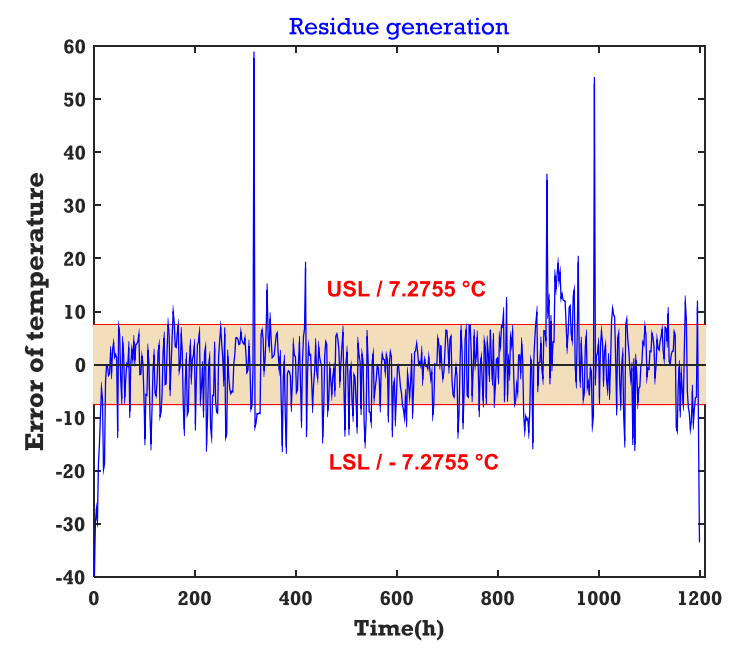

Fig. 19. The detection faults of (T2) with respect to a threshold

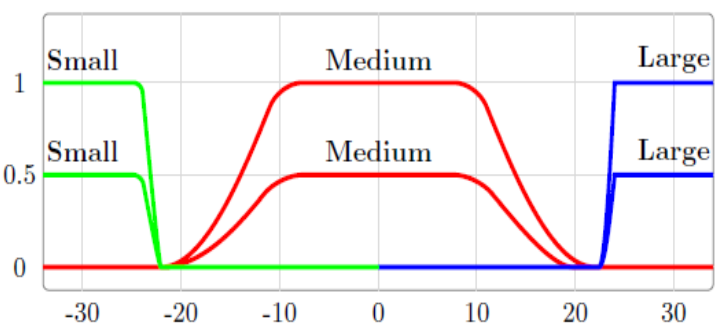

Fig. 20. Type-2 fuzzy sets of discharge pressure $P 2$ expert model faults detection

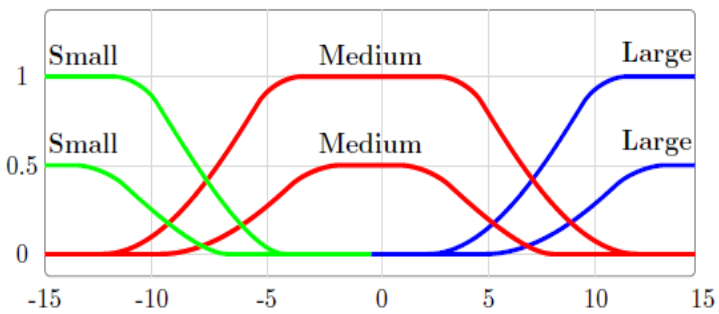

Fig. 21.Type-2 fuzzy sets of discharge temperature $T 2$ expert model faults detection

20 faults are detected related to the discharge pressure output P2, 12 faults were accurate during the first 12 hours and 8 faults during the time interval from 968 hours to 1142 hours, regarding the discharge temperature output $\mathrm{T} 2$, around 400 faults are detected, these faults have been occurred during almost the operating time along the interval time from 1 hour to 1208 hours.

The detection faults of the discharge pressure and the discharge temperature respectively are shown in Fig. 22 and Fig. 23 based on 2D thermal card, where the red color symbolizes the detected fault and each fault is represented by stem.

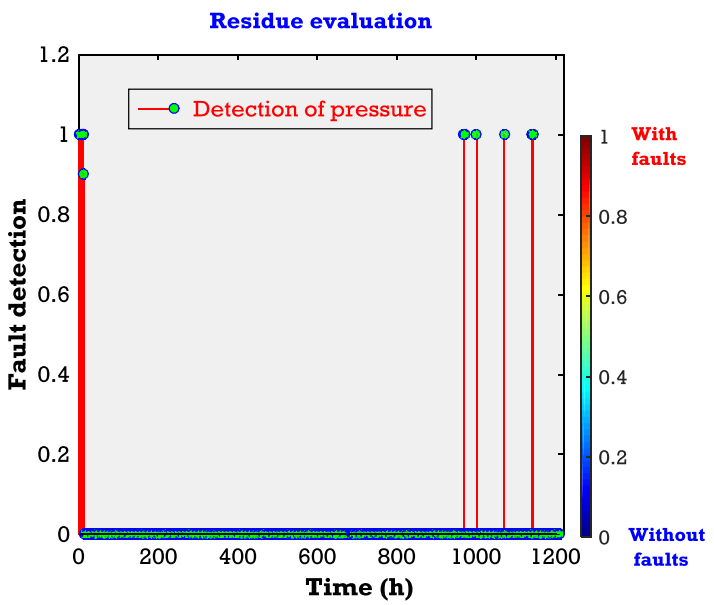

Fig. 22. The fault evaluation of ( $P 2)$ gas compressor system examined

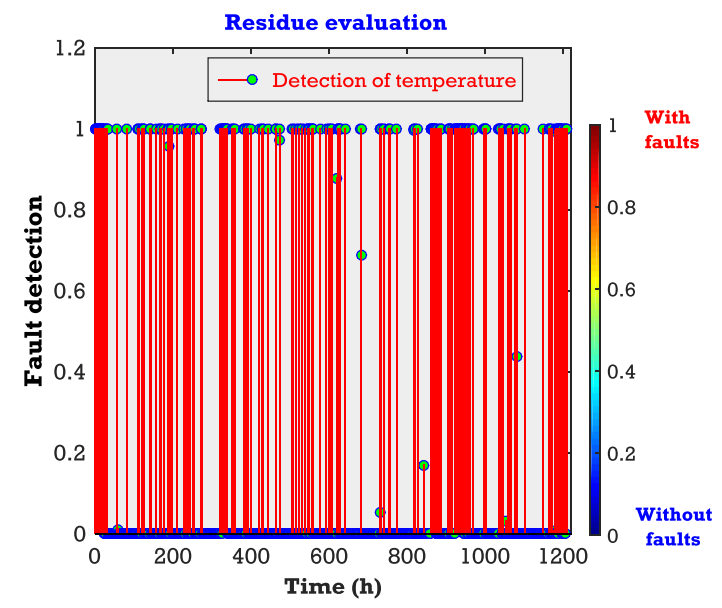

Fig. 23. The fault evaluation of (T2) gas compressor system examined

After the faults detection based on the output signals of the centrifugal gas compressor T2 and P2 through the proposed faults detection setup, a maintenance schedule is required for the system to identify the nature of the faults, the resulting damages and the affected components, where the main aim is to ensure the required change and reparation in the system to restart the system operating mode again.

During the experimental study, the system was completely opened for performing the maintenance, checking the inside body of the studied system and for the validation of the proposed faults diagnosis and detection presented in this paper and its accuracy for the determination and assessment of the fault levels. Indeed, after careful examination, the damage observed and realized in the components of the BCL 505 gas compressor are presented in the following figures:

$\checkmark$ scratch on the blades of the impeller1, as shown in Fig. 24 
$\checkmark$ light streaks at the spacers level, as shown in Fig. 25.

$\checkmark$ o-ring joints defects, as shown in Fig. 26.

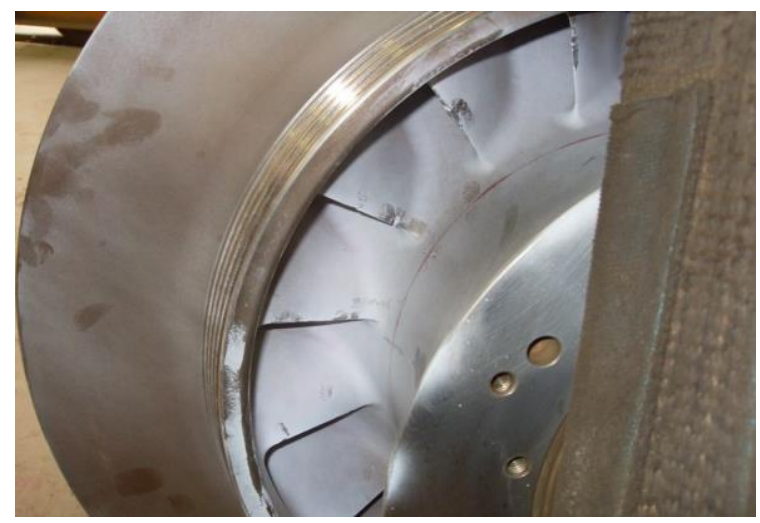

Fig. 24. The status of scratch on the blades of the impeller1.

\subsection{Forecasting the remaining time}

In this section, it is assumed that the centrifugal gas compressor is operating under faulty mode. In this case, the proposed approach of faults diagnosis and detection needs to be improved. It means that depending on the level and quality of faults, the safety time for which the studied system can continue its operation mode before it falls completely in failure and reaches the operating interruption. In order to achieve this important required task, the ARIMA model is used to know the evolution of both temperature and pressure with respect to time, to know when the centrifugal gas compressor be out of control (damage).

The ARIMA model and the interpolation algorithm techniques [37, 38] are highly used in speech signal processing and in statistics (time series), as well as in the deterministic and random case, which will be the only one considered in this work. The ARIMA model is used to predict the value of a discrete-time signal at a defined instant as a function of its past or all its future, the main aim of implementation of this algorithm is to predict the time at which the system reaches the stage of damage or danger.

After viewing and identifying the affected parts in the centrifugal gas compressor system, the below Table 2 confirm the results obtained from the proposed setup for the faults detection. In comparison with the constructor operating system documents, it can be said that the obtained results are within the norms given by the constructor.

From the results shown in Figures Figs. 27 to 29 , for the discharge temperature T2 output, the time between the alarm warning and the time of the system to be out of control is 60 hours. For the discharge pressure P2 and based on Figures. Figs. 30 to 32 , the time between the alarm warning and the time of the system to be out of control is 75 hours.

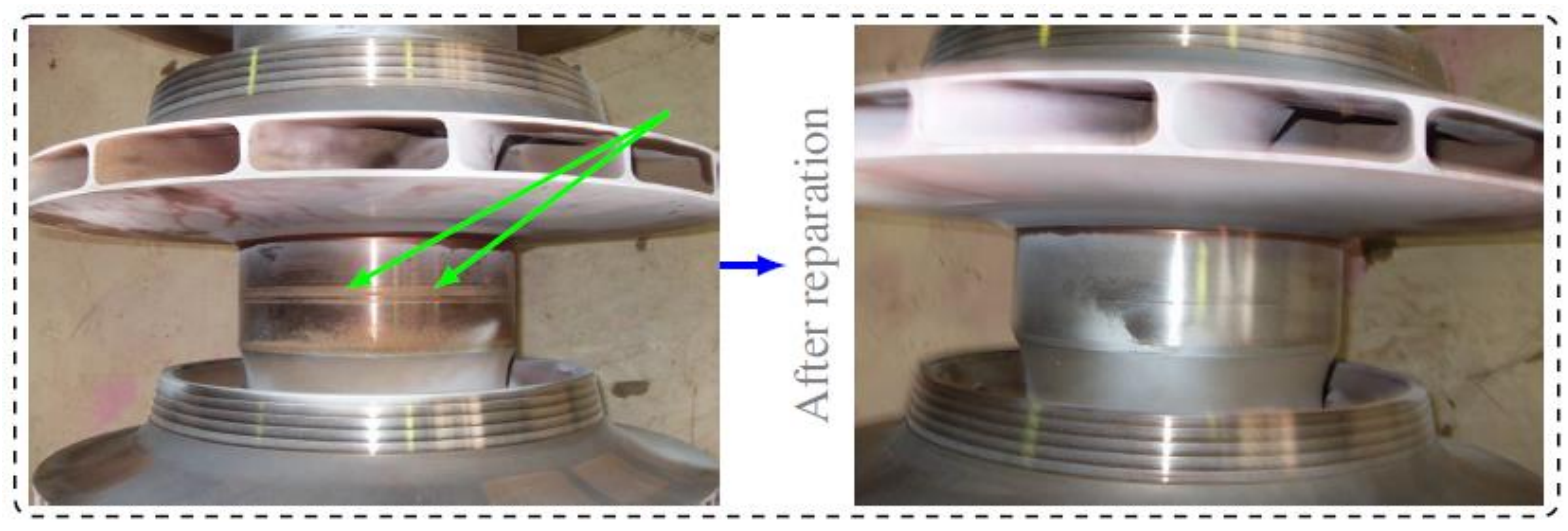

Fig. 25. The status of light streaks at the spacers level and after reparation.

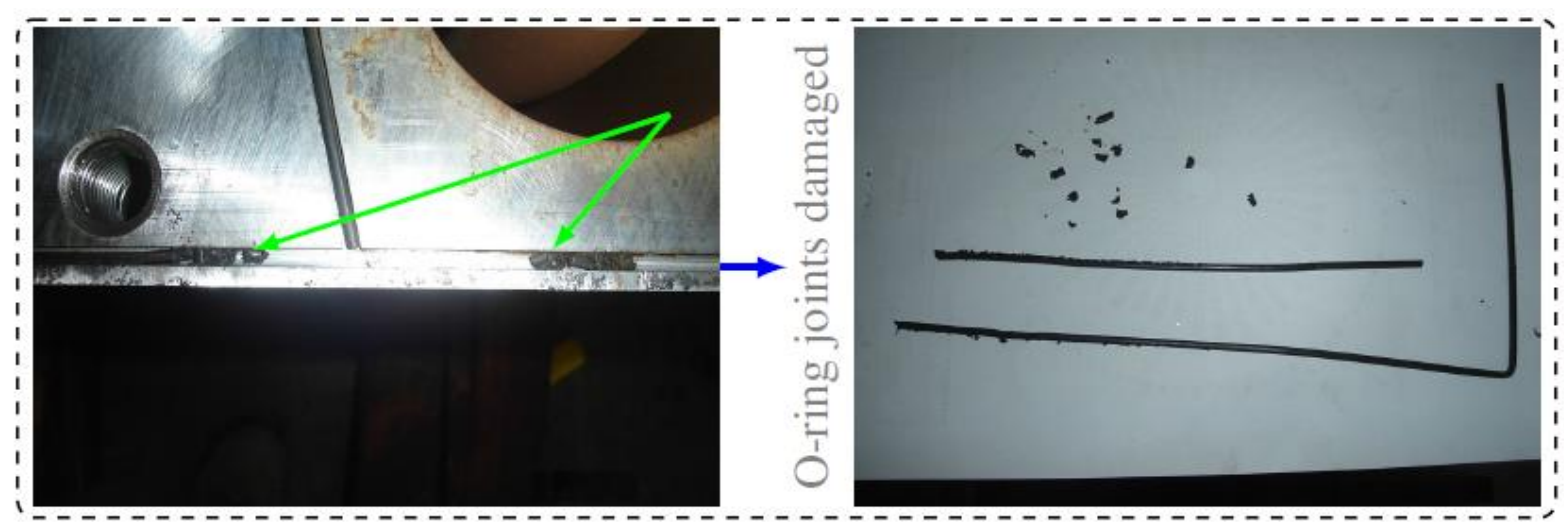

Fig. 26: The status of O-ring joints defective. 
Table.2. Faults identification and classification

\begin{tabular}{|c|c|c|c|}
\hline Faults & Outputs & Signal & Faults identification \\
\hline \multirow{2}{*}{$F_{1}$} & $\mathrm{P} 2$ & & \multirow{2}{*}{$\begin{array}{l}\text { - Distortions and } \\
\text { incision in the level } \\
\text { of blades }\end{array}$} \\
\hline & $\mathrm{T} 2$ & & \\
\hline \multirow{2}{*}{$F_{2}$} & $\mathrm{P} 2$ & & \multirow{2}{*}{$\begin{array}{l}\text { - O-ring joints } \\
\text { defective } \\
\text { - Friction between } \\
\text { the blades and } \\
\text { wheel-space }\end{array}$} \\
\hline & $\mathrm{T} 2$ & & \\
\hline \multirow{2}{*}{$F_{3}$} & $\mathrm{P} 2$ & & \multirow{2}{*}{$\begin{array}{l}\text { - looseness in screws } \\
\text { - O-ring joints } \\
\text { defective }\end{array}$} \\
\hline & $\mathrm{T} 2$ & & \\
\hline & & & \\
\hline
\end{tabular}

These results mean that the safety time for the system to operate under the detected faults until falling into danger or damage is 60 hours, beyond this time the safety of the whole system is not guaranteed. Thus, through these predictions, the system damages and its maintenance time loss can be avoided, furthermore, the system can be isolated in time from critical danger. It can be said from the obtained results that the proposed and improved faults diagnosis and detection approach in this paper can improve the operation of centrifugal gas compressor system and guarantee its production continuity within the whole installation.

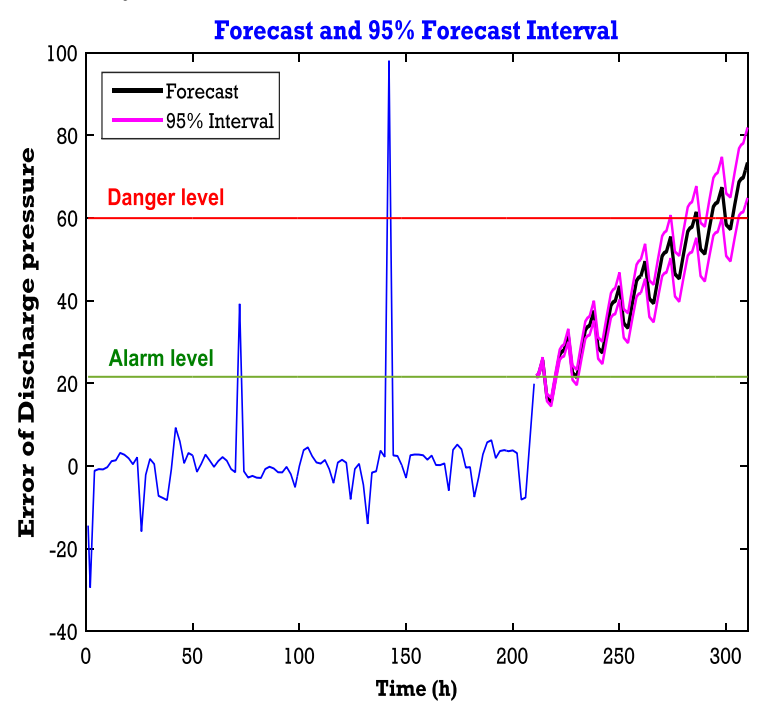

Fig. 27. Forecasting time level danger of T2

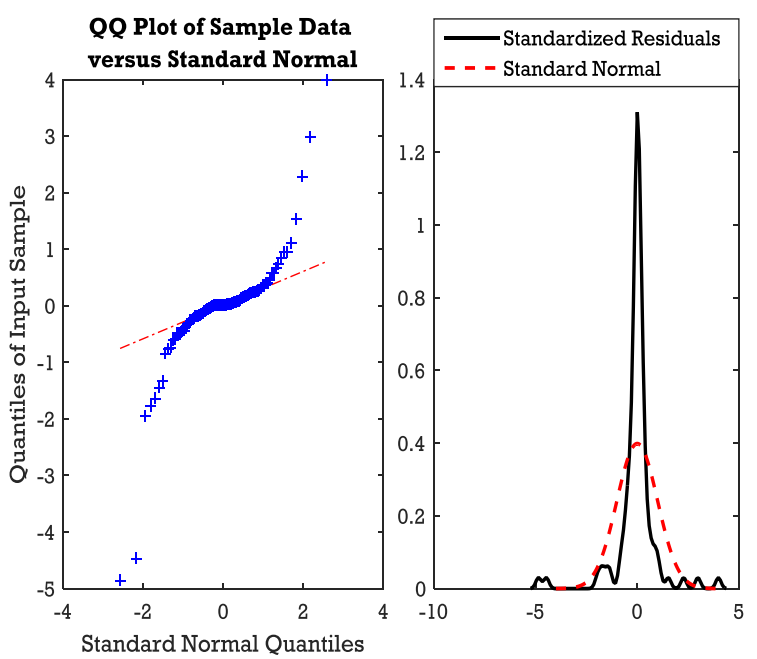

Fig. 28. The quantile-quantile plot (QQ-plot) and kernel density estimate of $\mathrm{T} 2$
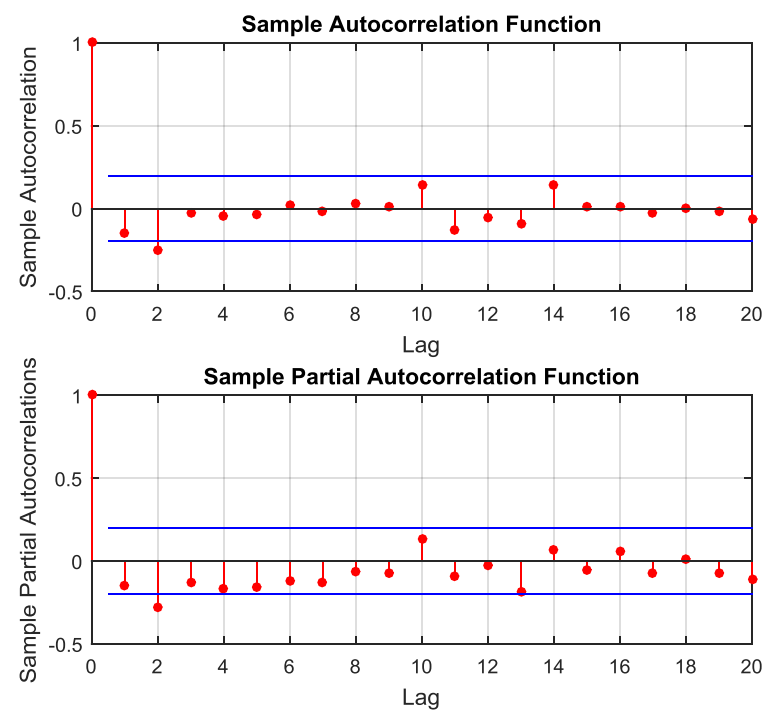

Fig. 29. Sample/and partial autocorrelation functions of $\mathrm{T} 2$

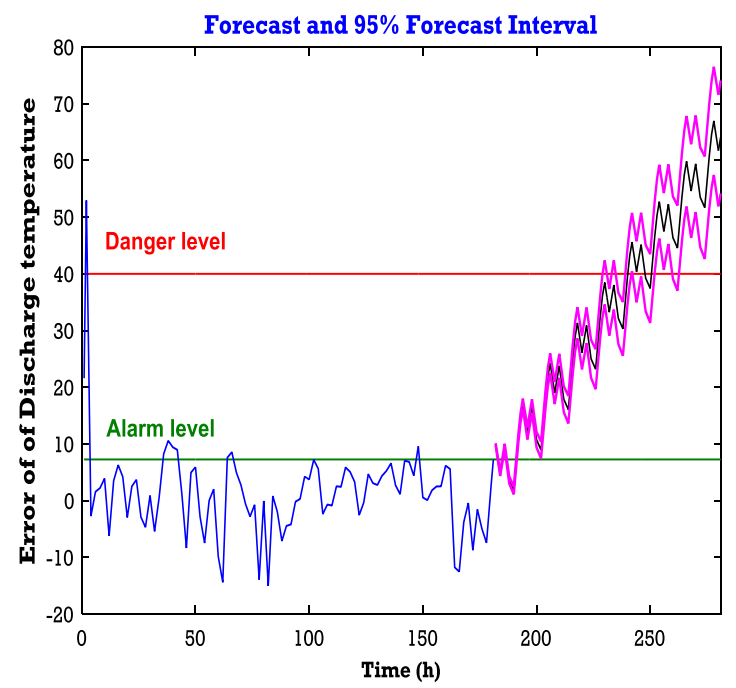

Fig. 30. Forecasting time level danger of P2 

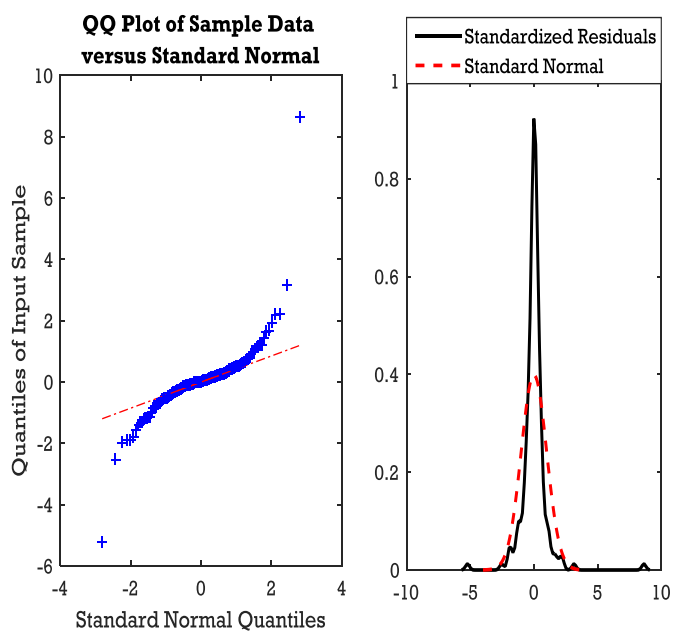

Fig. 31. The quantile-quantile plot (QQ-plot) and kernel density estimate of $\mathrm{P} 2$
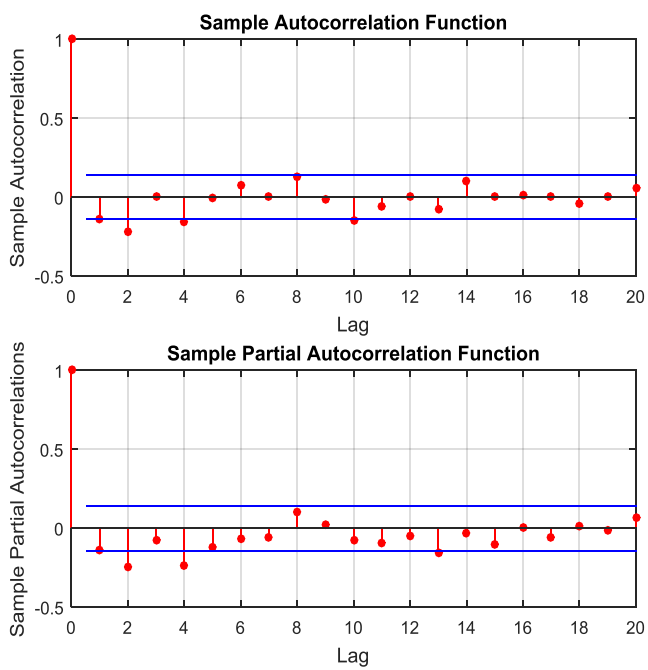

Fig. 32. Sample/and partial autocorrelation functions of $\mathrm{P} 2$

\section{ECONOMIC STUDY}

\subsection{Reparation costs}

During the first phase, within the time interval from 0 to 1000 hours, the centrifugal gas compressor operates in healthy operating mode, consequently the value of the faults detection system indicates "0" Fig. 33. During the second phase, along the time interval from 1000 hours to 2209 hours, the centrifugal gas compressor operates under intermittent faulty state. Hence, the faults detection system indicates " $1 "$ as the measured signal for faults detection pass the threshold aforementioned Fig. 33. It is important to clarify that the faults occurrence in percentage rises to $38.9776 \%$ at the end of the second phase, this percentage is calculated based on the number of zeros and ones obtained by the faults detection system during the second phase as shown in Fig. 33. this percentage value means that the performances of the centrifugal gas compressor are decreased, due to the increase of the temperature $\mathrm{T} 2$ and the decrease of the output pressure $\mathrm{P} 2$ as shown in Fig. 33 Consequently, at the end of the second phase, the machine is stopped to perform the maintenance of the whole system, not only the studied compressor. This operation is performed during the time of 744 hours ( 31 days) where the main aim is to achieve all the required reparations. On the other side, the total repair cost was estimated at $357,000 €$ (this maintenance is performed to all the equipment without the intervention of the manufacturer or an external company). It can be seen clearly that the reparation cost increases as the reparation time increase as shown in Fig. 33. After the reparation, the centrifugal gas compressor is restarted up and connected to the installation to work with a fully capacity again.

\subsection{Benefits}

When the intervention of reparation is performed at the time where the percentage of the fault occurrence reaches $10 \%$ based on the proposed diagnostic and faults detection system, the cost of reparation will be approximately $96,267 €$, furthermore the reparation period will take approximately 204 hours ( 8 days and a half day). In this case it can be said that the proposed diagnostic system for early faults detection is economically efficient, where the reparation intervention of defects will be done in time, more faster and with reduced financial burdens.

\section{CONCLUSION}

A robust faults diagnosis and detection approach is proposed in this paper and applied on the centrifugal gas compressor BCL 505 based on experimental data obtained on site from the measurement of the real time acquisition control system (ACS). The main purpose of the proposed approach is to improve the energy efficiency by improving the operating mode and the monitoring performance of the BCL 505 centrifugal gas compressor used in gas transportation station and studied in this paper.

This proposed faults diagnosis and detection approach is a combination of the two faults detection and isolation (FDI) approaches that are mainly based on the optimal identified healthy parametric equivalent model, the Kalman observation system, and the intelligent expert fuzzy type-2 system. Whereas the Kalman filer is used to obtain the estimation of the output signals and to ensure the robustness against the eventual disturbances and noise contained in the measured output signals from the DCS. The output of the optimal identified parametric model and the estimated output based on real time inputs are used to generate the current residues on real time. In this paper this residues are used through the expert 


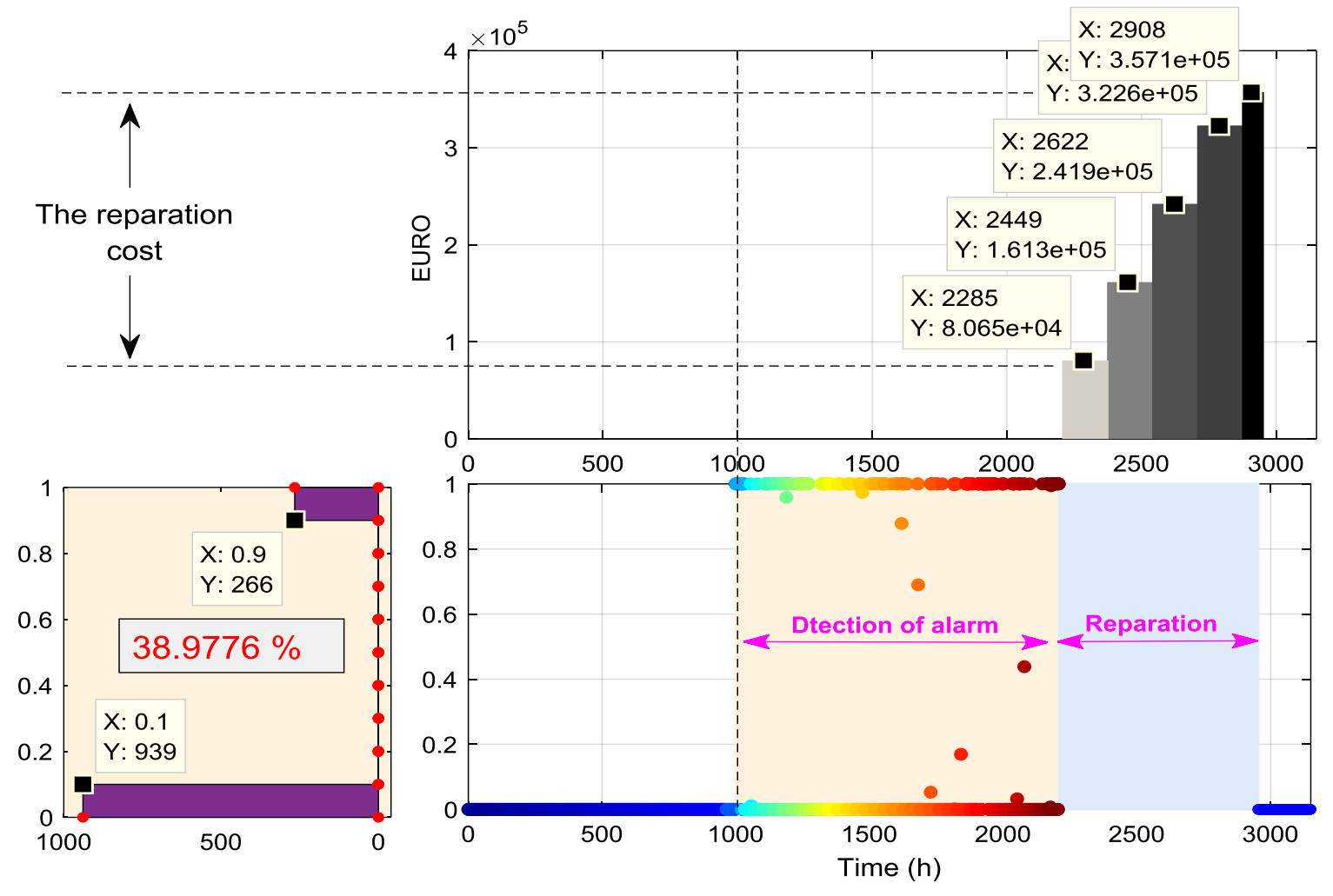

Fig. 33. The diagnosis approach impact on the financial cost

system which was designed based on the type-2 fuzzy logic to ensure the faults diagnosis and detection in real time, this expert system achieves this main task with the help of the calculated and defined limits of the threshold defining the degree of the faults and its level of damage and risk on the studied centrifugal gas compressor BCL 505 system. Furthermore, the prediction of the remaining time before the failure of the studied machine is investigate based on the autoregressive integrated moving average (ARIMA) model, where the main aim is to avoid the an expected failure and to provide an accurate maintenance schedule. It can be concluded that the proposed approach of faults diagnosis and detection which has been applied on the centrifugal gas compressor BCL 505 posses several advantage such as the decrease of the reparation time, avoiding the system form an expected operating risks and reducing the maintenance costs. These advantages affects positively the energy efficiency of the whole studied system and therefore increase the stability of the production, on the operation mode of the studied system and improves the overall studied system reliability and its robustness against the eventual faults.

Finally, it can be said that the proposed faults diagnosis and detection approach investigated in this paper is a promising approach which can be applied for different heavy industrial systems to improve their efficiency, especially in the area of petrol and oil industrial applications such as the gas turbine, the turbo-alternator, the turbo gas compressor...etc, where the main aim is to improve their dynamic behaviours, their operation mode, their reliability, their economics and their efficiency.

- The memberships functions of the fuzzy type 2 expert-system may be are not the optimal ones, we can use the PSO or Genetic Algorithms to select the optimal ones, as further studies.

2- The modeling of the studied system can be obtained based on Artificial Neural Network (ANN) or ANFIS algorithms for obtained the model that exactly represents all the dynamics behavior of the studied system.

\section{APPENDIX}

Table 3: General performance of centrifugal gas compressor BCL 505

\begin{tabular}{lc}
\hline Stages & $1-5$ \\
Maximum discharge pressure & $123 \mathrm{~kg} / \mathrm{cm}^{2}$ \\
Maximum discharge temperature & $121{ }^{\circ} \mathrm{C}$ \\
Efficiently -\% & $73 \%$ \\
Speed & 3000 to $20000 \mathrm{rpm}$ \\
Compressed gas & LNG \\
\hline
\end{tabular}

The process and measurement noise covariance matrices $\mathrm{Q}$ and $\mathrm{R}$, respectively have been selected

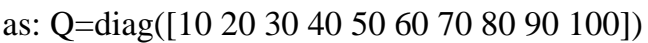

$\mathrm{R}=\operatorname{eye}(2)$ 


\section{REFERENCES}

1. Dai X, Gao Z, Breikin T, Wang H. Disturbance attenuation in fault detection of gas turbine engines: A discrete robust observer design, IEEE Transactions on Systems Man and Cybernetics Part C. Applications and Reviews, 2009;39(2):0-239. http://dx.doi.org/10.1109/TSMCC.2008.2005845

2. Hafaifa A, Guemana M, Daoudi A. Vibrations supervision in gas turbine based on parity space approach to increasing efficiency. Journal of Vibration and Control, 2015;21(8):1622-1632. https://doi.org/10.1177/1077546313499927

3. Bahareh P, Nader M, Khashayar K. Sensor fault detection, isolation, and identification using multiplemodel-based hybrid kalman filter for gas turbine engines. IEEE Transactions on Control Systems Technology, 2015; 24(4): 1184-1200. http://dx.doi.org/10.1109/TCST.2015.2480003

4. Hadroug N, Hafaifa A, Kouzou A, Chaibet A. Faults detection in gas turbine using hybrid adaptive network based fuzzy inference systems. Diagnostyka, 2016; 17(4): 3-17.

5. Bassily H, Lund R, Wagner J. Fault detection in multivariate signals with applications to gas turbines. IEEE Transactions on Signal Processing, 2009; 57(3): 835-842.

6. Isermann R. Fault-diagnosis systems: an introduction from fault detection to fault tolerance. SpringerVerlag Berlin Heidelberg, 2006.

7. Bailey MB, Kreider JF. Creating an automated chiller fault detection and diagnostics tool using a data fault library. ISA Transactions, 2003; 42:485-495.

8. Du Z, Domanski PA, Vance PW. Effect of common faults on the performance of different types of vapor compression systems. Applied Thermal Engineering, 2016;98:61-72. https://doi.org/10.1016/j.applthermaleng.2015.11.108

9. Yang Z, Shengwei W, Fu X. A statistical fault detection and diagnosis method for centrifugal chillers based on exponentially-weighted moving average control charts and support vector regression. Applied Thermal Engineering, 2013; 51:560-572. http://dx.doi.org/10.1016/j.applthermaleng.2012.09.0 $\underline{30 .}$.

10. Tsoutsanis E, Meskin N, Benammar M, Khorasani K. A component map tuning method for performance prediction and diagnostics of gas turbine compressors. Applied Energy, 2014;135(24):572585.

http://dx.doi.org/10.1016/j.apenergy.2014.08.115.

11. Joly R, Ogaji S, Singh R, Probert S. Gas-turbine diagnostics using artificial neural-networks for a high bypass ratio military turbofan engine. Applied Energy, 2004;78(4):397-418.

12. Shu Lin, Chunjie Yang, Ping Wu, Zhihuan Song. Active surge control for variable speed axial compressors, ISA Transactions 2014;53:1389-1395. https://doi.org/10.1016/j.isatra.2013.12.031

13. Tayarani-Bathaie SS, Khorasani K. Fault detection and isolation of gas turbine engines using a bank of neural networks. Journal of Process Control. 2015;36(12):22-41. https://doi.org/10.1016/i.jprocont.2015.08.007

14. Nozaria HA, Shoorehdelib MA, Simanic S, Banadakia HD. Model-based robust fault detection and isolation of an industrial gas turbine prototype using soft computing techniques. Neurocomputing,
2012; 91(16):29-47.

https://doi.org/10.1016/j.neucom.2012.02.014

15. Hafaifa A, Laroussi K, Laaouad F. Robust fuzzy fault detection and isolation approach applied to surge in centrifugal compressor modeling and control. Fuzzy Information and Engineering, 2010; 2(1):49-73.

16. Hafaifa A, Laaouad F, Laroussi K. Fuzzy approach applied in fault detection and isolation to the compression system control. Studies in Informatics and Control (SIC), 2010;19(1):17-26.

17. Hafaifa A, Laaouad F, Laroussi K. Fuzzy logic approach applied to the surge detection and isolation in centrifugal compressor. Automatic Control and Computer Sciences, 2010; 44(1):53-59.

18. Ogaji S, Marinai L, Sampath S, Singh R, Prober S. Gas-turbine fault diagnostics: a fuzzy-logic approach. Applied Energy, 2005;82(1):81-89.

19. Sahar Rahimi Malekshan, Mahdi Aliyari Shoorehdeli, Mostafa Yari. Industrial gas turbine compressor fouling detection based on system identification methods, neural networks and experimental data, 2017:709-714. https://doi.org/10.1109/IranianCEE.2017.7985130

20. Kenyon AD, Catterson VM, McArthur SDJ, Twiddle J. An agent-based implementation of hidden markov models for gas turbine condition monitoring. IEEE Transactions on Systems, Man, and Cybernetics: Systems, 2014; 44(2):186-195.

21. Sayyid Mahdi Alavinia. Surge avoidance in gas compressor via fault diagnosis. IEEE International Conference on Electrical, Computer and Communication Technologies (ICECCT), 2015:1-9. https://doi.org/10.1109/ICECCT.2015.7226005

22. Saavedra I, Bruno JC, Coronas A, Thermodynamic optimization of organic rankine cycles at several condensing temperatures: case study of waste heat recovery in a natural gas compressor station. Proceedings of the Institution of Mechanical Engineers Part A Journal of Power and Energy, 2010; 224(7):917-930.

23. Maleki S, Bingham C, Zhang Y. Development and realization of changepoint analysis for the detection of emerging faults on industrial systems. IEEE Transactions on Industrial Informatics, 2016;12(3):1180-1187.

24. Tabkhi F, Pibouleau L, Hernandez-Rodriguez G, Azzaro-Pantel C, Domenech S. Improving the performance of natural gas pipeline networks fuel consumption minimization problems. AIChE Journal, 2010; 56(4): 946-964.

25. Mohamadi B, Mohamad, Tabkhi F, Sargolzaei J. Exergetic approach to investigate the arrangement of compressors of a pipeline boosting station. Energy Technology, 2014;2(8):732-741.

26. Hafaifa A, Laaouad F, Laroussi K. Fuzzy modelling and control for detection and isolation of surge in industrial centrifugal compressors. Automatic Control Journal of the University of Belgrade, 2009; 19(1): 19-26.

27. Nail B, Kouzou A, Hafaifa A, Chaibet A. Parametric identification and stabilization of turbo-compressor plant based on matrix fraction description using experimental data. Journal of Engineering Science and Technology, 2018; 13(6):1850-1868.

28. Hadroug N, Hafaifa A, Kouzou A, Chaibet A. Dynamic model linearization of two shafts gas turbine via their input/output data around the 
equilibrium points. Energy, 2017; 120(2):488-497. https://doi.org/10.1016/j.energy.2016.11.099

29. Jiang W, Khan J, Dougal R A. Dynamic centrifugal compressor model for system simulation. Journal of Power Sources, 2006; 158(2):1333-1343.

30. Barszcz T, Zimroz R, Urbanek J, Jabłoński A, Bartelmus W. Bearings fault detection in gas compressor in presence of high level of non-gaussian impulsive noise. Key Engineering Materials, 2013; 569-570(2):473-480. https://doi.org/10.4028/www.scientific.net/KEM.569570.473

31. Alavinia SM, Khosrowjerdi MJ, Sadrnia MA, Kheiri $\mathrm{H}$, Fateh MM. An algebraic approach to fault detection for surge avoidance in turbo compressor. Journal of Engineering for Gas Turbines and Power, 2014; 137(2):1-8. https://doi.org/10.1115/1.4028370

32. Mohtar H, Chesse P, Chalet D. Describing uncertainties encountered during laboratory turbocharger compressor tests. Experimental Techniques. 2012; 36(5):1-9.

33. Bachir N, Abdellah K, Ahmed H. Robust block roots assignment in linear discrete-time sliding mode control for a class of multivariable system: gas turbine power plant application. Transactions of the Institute of Measurement and Control, 2018:1-17. https://doi.org/10.1177/0142331218774615

34. Akroum M, Hariche K. An optimal instrumental variable identification method for $1 \mathrm{mfd}$ models. Studies in informatics and control, 2008;17(4): 361372.

35. Ozek MB, Akpolat ZH. A software tool: Type-2 fuzzy logic toolbox. Computer Applications in Engineering Education, 2008; 16(2):137-146.

36. Manceur M, Essounbouli N, Hamzaoui A. Secondorder sliding fuzzy interval type-2 control for an uncertain system with real application. IEEE Transactions on Fuzzy Systems, 2012;20(2):262-275.

37. Box GEP, Jenkins GM, Reinsel GC, Ljung GM. Time series analysis: forecasting and control, 5th edition. Wiley Series in Probability and Statistics, Wiley, 2015.

38. Ting Z, Li L, Xinli Z, Yingkang S, Wenwu S. Timeseries approaches for forecasting the number of hospital daily discharged inpatients. IEEE Journal of Biomedical and Health Informatics, 2015; 21(2):515526. https://doi.org/10.1109/JBHI.2015.2511820

39. Taskin A, Kumbasar T. IEEE symposium series on computational intelligence (SSCI) - cape town, South Africa. IEEE symposium series on computational intelligence - an open source matlab/simulink toolbox for interval type-2 fuzzy logic systems, 2015: 15611568.

40. Ljung L. System Identification: Theory for the User, 2nd Edition, Prentice Hall, 1999.

Received 218-10-23

Accepted 2019-04-23

Available online 2019-05-10
Mr. Bachir NAIL received his License and master's degrees in electrical engineering, Automatic control from Ziane Achour University Djelfa, Algeria in 2013 and 2015 respectively. $\mathrm{He}$ is currently a Ph.D. student at Applied Automation and Industrial Diagnostics Laboratory, Faculty of Sciences and Technology, University of Djelfa, Algeria. His current research interests include, modeling and control of dynamic systems, estimation theory, fault tolerance control and fault detection theory, matrix polynomial theory, Multivariable systems, Optimization approaches. $\mathrm{He}$ has participated in many workshops and published many national/international conferences and journals papers within his domain of interest.

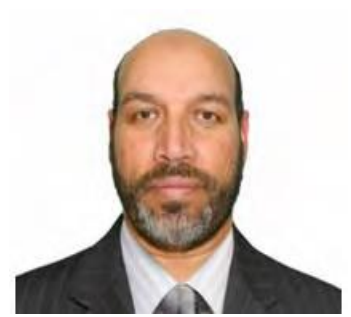

Prof. Abdellah KOUZOU (IEEE Senior memebr \& IACSIT Senior member, IFAC,IAENG \& IISRO member, IEEE-HKN Alumni Member) was born in Djelfa, Algeria in 1964. $\mathrm{He}$ received the State Engineer degree, the Magister, the $\mathrm{PhD}$, and the Habilitation from University of Tiaret, Algeria, University of Boumerdes, Polytechnics Superior National School respectively. He has been a researcher with Technische Universität of Muenchen in Germany form 2010, 2011 and 2012. He is a collaborator collaborator researcher at Texas A\&M University at Qatar. $\mathrm{He}$ is an associate professor with the faculty of Sciences and Technology at Djelfa university in Algeria, where he was the president of the Scientific council of the faculty since June 2014. Dr. Kouzou has participated in several research projects and has led several research projects. he was the ex-dean of the faculty of Sciences and Technology at Djelfa university in Algeria.

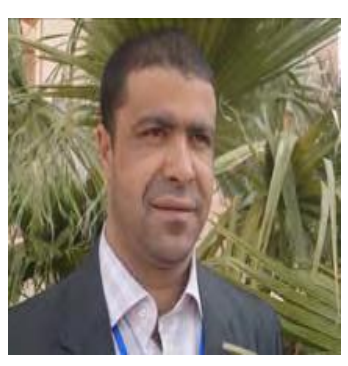

Prof. Ahmed HAFAIFA is the founder of the Applied Automation and Industrial Diagnostic Laboratory at the University of Djelfa. He is the supervisor of many $\mathrm{PhD}$ Students and he is the coordinator of several industrial research projects within the applied automatic diagnostics and reliability of industrial systems. His research area of interests includes the modelling and control in industrial systems, the diagnosis and new reliability engineering, fault detection and isolation in industrials process, intelligent system based on fuzzy logic and neural networks. He is active as an expert in several national and internationals committees and collaboration research activities. He has participated in several international research 


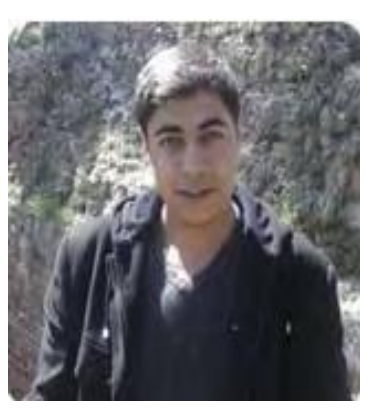

Dr. Naji HADROUG was born on 18/12/1989 in Hassi Bahbah, Djelfa, Algeria. He is a member of the Applied Automation and industrial diagnostics laboratory, university Djelfa. he is actual working toward his $\mathrm{PhD}$ thesis. His main researches are on the NeuroFuzzy fault tolerant control applied on a gas turbine. His thesis focuses on the development of new methods and tools in control fault tolerant for industrial systems. He is author and co-author of several publications and conference papers.

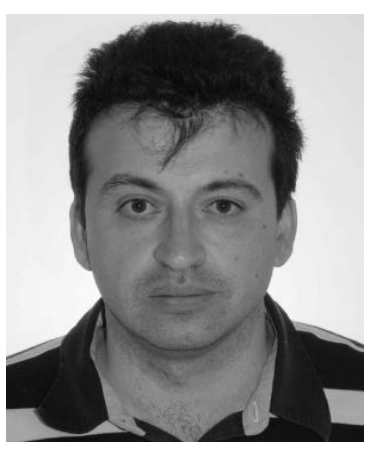

\section{Prof. Vicenç Puig}

received the Telecommunications Engineering degree and the Ph.D. degree in automatic control, vision, and robotics from Universitat Politècnica de Catalunya (UPC), Barcelona, Spain, in 1993 and 1999, respectively., $\mathrm{He}$ is a Professor with the Automatic Control Department, UPC, where he is also a Researcher with the Institut de Robòtica Informàtica Industrial. $\mathrm{He}$ is the Chair of the Automatic Control Department and the Head of the research group in Advanced Control Systems, UPC. He has developed important scientific contributions in the areas of fault diagnosis and fault tolerant control using interval and linear-parameter-varying models using set-based approaches. He has participated in over 20 European and national research projects in the last decade. He has also led many private contracts with several companies and has published over 100 journal articles and over 350 in international conference/ workshop proceedings. He has supervised over $15 \mathrm{Ph} . \mathrm{D}$. dissertations and over 40 master's theses/final projects., Prof. Puig is currently the Vice-Chair of the IFAC Safe process TC Committee 6.4 from 2014 to 2017 . He is the Chair of the Third IEEE Conference on Control and Fault-Tolerant Systems (Systol 2016) and the IPC Chair of the IFAC Safe process 2018. 